\title{
On multi-criteria chance-constrained capacitated single-source discrete facility location problems ${ }^{\text {th }}$
}

\author{
Ömer Burak Kınay ${ }^{\mathrm{a}, 1}$, Francisco Saldanha-da-Gama ${ }^{\mathrm{b}, \mathrm{c}}$, Bahar Y. Kara ${ }^{\mathrm{d}, *}$ \\ a Department of Management Sciences, University of Waterloo, Waterloo, ON, Canada \\ ${ }^{\mathrm{b}}$ Departamento de Estatística e Investigação Operacional, Faculdade de Ciências, Universidade de Lisboa, Campo Grande, Lisboa, 1749-016, Portugal \\ ' Centro de Matemática, Aplicações Fundamentais e Investigação Operacional, Faculdade de Ciências, Universidade de Lisboa, Campo Grande, Lisboa, \\ 1749-016, Portugal \\ ${ }^{\mathrm{d}}$ Department of Industrial Engineering, Bilkent University, Ankara, Turkey
}

\section{A R T I C L E I N F O}

\section{Article history:}

Received 24 May 2017

Accepted 8 February 2018

Available online 20 February 2018

\section{Keywords:}

Multi-criteria optimization

Discrete facility location

Single-sourcing

Chance-constraints

Humanitarian logistics

\begin{abstract}
A B S T R A C T
This work aims at investigating multi-criteria modeling frameworks for discrete stochastic facility location problems with single sourcing. We assume that demand is stochastic and also that a service level is imposed. This situation is modeled using a set of probabilistic constraints. We also consider a minimum throughput at the facilities to justify opening them. We investigate two paradigms in terms of multicriteria optimization: vectorial optimization and goal programming. Additionally, we discuss the joint use of objective functions that are relevant in the context of some humanitarian logistics problems. We apply the general modeling frameworks proposed to the so-called stochastic shelter site location problem. This is a problem emerging in the context of preventive disaster management. We test the models proposed using two real benchmark data sets. The results show that considering uncertainty and multiple objectives in the type of facility location problems investigated leads to solutions that may better support decision making.
\end{abstract}

(C) 2018 Elsevier Ltd. All rights reserved.

\section{Introduction}

A facility location problem consists of deciding where to locate one or several facilities in order to serve a set of demand points. Often, the goal is to minimize the total cost that includes establishing the facilities and supplying the demand. In a discrete setting there is a finite set of potential locations for the facilities; in the single-source variant of the problem, all the demand of a customer must be supplied from a single facility. The reader can refer to the book chapter by [20] for a synthesis of the most relevant work done on fixed-charge facility location problems that includes the single-source capacitated facility location problem as a particular case.

In this paper we investigate the single-source extension of the problem that emerges when (i) facilities are capacitated, (ii) demands are stochastic, and (iii) multiple objectives are to be jointly considered.

\footnotetext{
is This manuscript was processed by Associate Editor Dr. Joseph Geunes.

* Corresponding author.

E-mail address: bkara@bilkent.edu.tr (B.Y. Kara).

1 This research was initiated when the author was at Department of Industrial Engineering, Bilkent University, Ankara, Turkey.
}

Two well-known paradigms in multi-criteria optimization will be considered: vectorial optimization and goal programming. Concerning stochasticity, we assume that it can be captured mathematically via a set of probabilistic constraints.

In addition to proposing different modeling frameworks for a general problem, we investigate the relevance of such developments by applying the new models to a case study in the context of the so-called shelter site location problem, which is a problem emerging in the context of preventive disaster management. In this case, typically, a weight can be assigned to each facility with larger weights indicating larger suitability of the facilities according to their purpose. The specific objectives considered in the case study are the maximization of the minimum weight among the selected facilities; the maximization of the average weight among the selected facilities; the minimization of the average distance traveled by the customers to reach their assigned facility. The first objective may not appear as natural/intuitive as the other two. However, its relevance is justified by applications in which a focus is put on the least advantaged populations/customers. In such cases, the maximization of their "benefit" is a way for achieving a more "fair" system. We deepen this discussion in Section 4 when introducing the case study. 
Single-source (capacitated) facility location problems have been studied for many years; in fact, many references can be found such as those by $[5,12,16,22,27,37]$, just to name a few. A common aspect to all these works is the use of a cost-oriented objective functionto be minimized. Furthermore, demand is deterministic, i.e, it is known in advance and it is not subject to any sort of uncertainty.

The relevance of developing optimization models and solution techniques in the context of facility location under uncertain demand has been widely recognized by the scientific community. This is attested by the literature covering that aspect which, in turn, has encouraged new research directions to be explored. For additional information the reader can refer to [11] and to the references therein. In the particular case of problems with single sourcing, we refer to the works by [2,3], who study the so-called facility location problem with Bernoulli demands. This is a single-source capacitated facility location problem with unit-demand customers and uncertainty in demand. The objective is to minimize the total (expected) cost, which includes the setup cost for the facilities plus the expected service and outsourcing cost (outsourcing occurs when the installed capacity is not enough for handling the occurring demand). Bieniek [7] worked on the same setting but considering other probability distributions for the demand. In these works, the problems were formulated mathematically using a two stage stochastic programming modeling framework.

A different type of approach was proposed by [28]. In this case, the demand of a customer is measured in terms of the quantity of a commodity to be delivered. Again, the goal is to minimize the total cost for establishing the facilities and supplying the customers. The author considered a service level that is captured mathematically using probabilistic constraints. This is motivated by the fact that considering "hard" capacity constraints may be meaningless when demand is uncertain. Nevertheless, if the uncertain demand can be described using a probability law it is possible to consider probabilistic constraints stating that the probability of having capacity for supplying the occurring demand should be above some threshold exogenously defined.

Kinay et al. [26] also consider a single-source discrete facility location problem with stochastic demand. Again, a service level constraint is included in the optimization models proposed. However, the objective function is of a totally different nature: the goal now is to maximize the minimum weight among the installed facilities. The problem is motivated by an application in the context of humanitarian logistics calling for the potential locations to be previously assigned a numerical weight that summarizes quantitatively several relevant features. The maxmin objective considered in that work was initially introduced by [25] and raises an interesting discussion in the context of discrete capacitated single-source facility location problems. In fact, that objective is prone in terms of producing "plateaus" in the objective function space: one can easily find multiple optimal solutions. However, in practice, a decision maker does not look at these solutions as being "equally" good/optimal. Accordingly, there is room for considering other objectives that may guide the decision maker when the time comes for deciding among those alternative optima. The above aspects trigger the work done in the current paper.

The literature focusing on stochastic multi-criteria facility location problems is still scarce. Even if we consider deterministic facility location problems, [35] argue that there is still much room for investigating the topic. When it comes to the particular case in which we have single sourcing even less can be found. To the best of the authors' knowledge, the closest works focusing on that very specific type of problems are due to [13] who, focus on a bi-criteria single-allocation hub location problem and to [29] who integrated the three dimensions we are also considering in our work using a weighting approach rather than the multi-criteria approaches that we propose.
The contribution of the current paper to the literature is threefold: (i) to consider multiple objective functions in a discrete capacitated single-source facility location problem with probabilistic constraints; (ii) to discuss the use of several objectives of practical relevance that have not been often used in the context of discrete facility location problems; (iii) to present a case study showing that the analysis performed in this work leads to solutions that can better adjust to a real setting.

As already mentioned, in this paper we study two paradigms in terms of multi-criteria analysis: vectorial optimization and goal programming. In the first case, no hierarchy is associated with the objective functions to be considered. In the second case, a hierarchy is assumed for the objectives and thus, each objective can be optimized only after the ones higher in the hierarchy have been studied and considering the multiple optimal solutions obtained so far.

Concerning the use of vectorial optimization in discrete facility location we refer the reader to [34], who present references until 2005, to [35] with recent references and to the survey paper by [47]. Looking into these references we observe that not much work has been published that is related with multi-criteria discrete facility location problems and let alone when it comes to single-source problems. A notable exception is the paper by [15]. This is a work proposing an interactive procedure aiming at finding non-dominated solutions to a bi-criteria single-allocation facility location problem. In particular, the authors proposed a specially tailored approach for the auxiliary problem considered for finding non-dominated solutions.

The application that triggered the current work and that underlies the case study to be discussed may call for the use of a goal programming approach since it conveys a case in which we may easily find a hierarchy between different but relevant objectives. Looking into the literature, we were able to find two works considering the use of goal programming in the context of discrete facility location problems: these are the papers by [40] and [4]. Nevertheless, in both works, multiple sourcing is assumed. The closest work to what we are presenting in this paper is the work by [43], who considered fuzzy set theory for capturing vagueness and ambiguity that may emerge when considering qualitative criteria. Nevertheless it is worth pointing out that in fact the authors do not consider uncertainty in demand-as we do in the current work-but vagueness in the information required for defining the criteria to be used.

Last but not least, we note that although not much work can be found in terms of optimization models and tools for multi-criteria discrete facility location, the study of several objective functions in the context of location analysis in general is far from novel. In other words, although related literature have considered a wide variety of objective functions, a single objective is considered at a time, rather than using a multi-criteria approach. The interested reader can refer to the reviews provided by $[14,17,47]$, as well as to the book chapters by [34] and [35].

Current et al. [14] classify the objectives of relevance in facility location according to: (i) cost minimization, (ii) demand-oriented, (iii) profit maximization, and (iv) environmental-oriented. In turn, [17] classify the objectives as (i) pull, (ii) push, and (iii) balance objectives. [47] specify 9 often-used objectives in location analysis, each of which falling in one of the above categories.

In this paper, we specifically analyze three objective functions of relevance in humanitarian logistics, two of which based upon measuring the potential locations for opening the facilities with a weight previously determined according to several features. Accordingly, we consider the maximization of the minimum weight among the selected locations and the maximization of the average weight (across the selected locations). Additionally, we analyze an- 
other objective that is related with the overall distance traveled by customers.

The remainder of this paper is organized as follows: in Section 2 we discuss modeling aspects related with chanceconstrained single-source discrete facility location problems. In Section 3 we analyze the new objectives considered in this work and their inclusion in mathematical modeling frameworks using vectorial optimization and goal programming. In Section 4 we present a case study and provide some methodological specifications required by it. In Section 5 we report the results of extensive numerical experiments performed for evaluating the relevance of the models proposed. The paper ends with an overview of the work done.

\section{A modeling framework for stochastic single-source capacitated facility location problem}

The basic ingredients for a discrete single-source capacitated facility location problem include a set $I$ of potential locations for the facilities, a set $J$ of demand points, a value $q_{i}$ associated with each location $i \in I$ denoting the capacity of a facility if installed in that location and a value $d_{j}$ associated with each demand point $j \in J$ representing its demand. The decisions to make are twofold: the location of the facilities and the allocation of demand points to the open facilities. An optimization model can be formulated considering two sets of decision variables: for $i \in I, x_{i}$ is equal to 1 is facility $i$ is open and zero otherwise; for $i \in I$ and $j \in J, y_{i j}$ is equal to 1 if demand point $j$ is allocated to facility $i$ and zero otherwise. Accordingly, a generic optimization model for the problem can be written as follows (see, for instance, [16,24], and [20]):

minimize $f(\mathbf{x}, \mathbf{y})$,

subject to $\sum_{i \in I} y_{i j}=1, \quad j \in J$,

$\sum_{j \in J} d_{j} y_{i j} \leq q_{i} x_{i}, \quad i \in I$,

$x_{i} \in\{0,1\}, \quad i \in I$,

$y_{i j} \in\{0,1\}, \quad i \in I, j \in J$.

Function $f(\mathbf{x}, \mathbf{y})$ represents the objective function to be minimized with $(\mathbf{x}, \mathbf{y})=\left(\left(x_{i}\right)_{i \in I},\left(y_{i j}\right)_{i \in I, j \in J}\right)$ denoting the vector of decision variables; constraints (2) ensure that each demand point is allocated to one and only one facility whereas constraints (3) are the capacity constraints. These constraints also ensure that a demand point can be allocated to a facility only if the facility is open.

Finally, (4) and (5) define the domain of the decision variables. We note that the above model can be enhanced in terms of the bounds provided by linear relaxation by the inclusion of the socalled strong-model inequalities $y_{i j} \leq x_{i}, \quad i \in I, \quad j \in J$ (see, for instance, [22]).

As we detailed in the introductory section, in this work we focus on the situation where demands are stochastic, i.e., we assume that $\xi=\left(d_{1}, \ldots, d_{|J|}\right)$ is a random vector with a joint cumulative distribution function that we assume to be given in advance (e.g., estimated using historical data). In this case, the (hard) capacity constraints (3) are no longer well-defined. One possibility could be to state those constraints using the most conservative values for the demands. However, planning for the largest possible demands may render a too "fat" namely, if that "scenario" corresponds to a very unlikely "future". An alternative is to consider probabilistic constraints ensuring a pre-specified service level. Let us denote by $\gamma_{i}$ a user-defined threshold (or an upper bound) value of the probability of exceeding the capacity of plant $i$, once the plants to be opened are decided and the allocations are determined. A service level constraint adequate for replacing (3) is

$\mathbb{P}_{\xi}\left[\sum_{j \in J} d_{j} y_{i j} \leq q_{i} x_{i}\right] \geq 1-\gamma_{i}, \quad i \in I$.

The above constraints, which have been considered by other authors (e.g., [28] and [26]), lead to a generalization of the original model since they reduce to (3) when data are deterministic and all demand must be supplied (i.e., a service level equal to $100 \%$ ).

In addition to the above service level constraints, there are other ways for extending model (1)-(5). One aspect of relevance in some problems is the existence of a minimum throughput that justifies opening a facility. This has been discussed in the context of logistics applications by [32] and [33] and in a broader context by $[2,3]$. In the context of humanitarian logistics, this aspect may also be important as discussed by [25] and [26]. In the latter work, the authors propose mathematical expressions for modeling such conditions: they consider a minimum threshold denoted by $\beta$ for the utilization rate of a facility and include the following set of additional constraints in their optimization model:

$\mathbb{P}_{\xi}\left[\sum_{j \in J} d_{j} y_{i j} \geq \beta q_{i} x_{i}\right] \geq 1-\zeta_{i}, \quad i \in I$.

In the above constraints, $\zeta_{i}$ denotes the (exogenous) probability that the minimum threshold of shelter $i \in I$ is not satisfied (recall that demands are random).

Since we are working with a problem emerging in the context of facility location with single sourcing we also consider a feature of practical relevance in these problems, which is discussed in the paper by [19] as well as in some references therein: the need for imposing the so-called closest assignment constraints in single-source facility location problems (depending on the application considered). These constraints are used to model situations in which the demand points should be assigned to the closest facility among those selected to operate. As we will see in the case study presented in Section 4, these constraints help to mimic the behavior of people when searching for the closest facility that can supply their needs. Several alternatives have been proposed in the literature for modeling mathematically the closest assignment constraints. Possibly, the best-known conditions are the original inequalities proposed by [39] and [45]. Kilcı et al. [25] and Kinay et al. [26] considered them in the context of humanitarian logistics to mimic the behavior of the victims of a disaster when looking for help. Espejo et al. [19] showed that [45]'s closest assignment constraints (WF) dominate the ones introduced by [39] (RR). Hence, they suggest using the former, i.e.:

$\sum_{s=r+1}^{|I|} y_{i_{j}(s), j}+x_{i_{j}(r)} \leq 1, \quad j \in J, r=1, \ldots,|I|-1$.

In the above expression $i_{j}(r)$ stands for the $r$-th closest candidate facility location to demand point $j \in J, r=1, \ldots,|I|$. For every demand point $j \in J$, one can easily find the above sorted facilities by sorting non-decreasingly the distances, say $\ell_{i j}$, between the potential locations $i \in I$ and customers $j \in J$.

In synthesis, the starting point for our study is the generic model

minimize

subject to

$(2),(4)-(8)$,

$$
y_{i j} \leq x_{i}, \quad i \in I, j \in J .
$$

We note the need for including the inequalities (9) in the absence of inequalities (3). 
Before discussing a multi-criteria setting it is important to deepen the analysis of the probabilistic constraints (6) and (7) because they raise some mathematical difficulties when it comes to tackling the model. As we explain next, it is possible to take advantage of the fact that we are working with a facility location problem in order to overcome such difficulties.

We first note the well-known fact that in many real-world facility location problems the number of demand points is quite large, namely when compared to the number of facilities that are eventually open (see, e.g., the discussion presented by [9]). From a demand allocation perspective, this fact leads to "many" demand points being assigned to each open facility. Furthermore, often, demand points are themselves the result of some previous aggregation. Accordingly, we often observe that the total demand served by a facility is actually the sum of "many small demands".

A second aspect to consider is that under uncertainty, demands can often be assumed independent. This means that instead of working with the joint cumulative probability function associated with the underlying random vector $\xi=\left(d_{1}, \ldots, d_{|J|}\right)$ we can directly consider the marginal cumulative distribution functions associated with the random variables $d_{1}, \ldots, d_{|J|}$. This typically simplifies the analysis.

Although starting from a general setting, the above remarks allow us to invoke the Central Limit Theorem, thus deriving an approximate model for the problem we are investigating. Denoting by $\mu_{j}$ and $\sigma_{j}^{2}$ the expected value and variance of $d_{j}(j \in J)$, by $z_{\alpha}$ the $\alpha$-quantile of the standard normal distribution and defining $v_{i}=\frac{\sqrt{\sum_{j \in J} \sigma_{j}^{2} y_{i j}}}{\sqrt{\sum_{j \in J} \sigma_{j}^{2}}}, i \in I$, (6) and (7) can be altogether replaced by the following deterministic constraints (the reader should refer to [28] and [26] for all details):

$\sum_{j \in J} \frac{\mu_{j}}{\Gamma} y_{i j}+z_{1-\gamma_{i}} v_{i} \leq \frac{q_{i}}{\Gamma} x_{i}, \quad i \in I$

$\sum_{j \in J} \frac{\mu_{j}}{\Gamma} y_{i j}+z_{\zeta_{i}} v_{i} \geq \frac{\beta q_{i}}{\Gamma} x_{i}, \quad i \in I$,

$v_{i}^{2}=\sum_{j \in J} \frac{\sigma_{j}^{2}}{\Gamma^{2}} y_{i j}, \quad i \in I$,

$0 \leq v_{i} \leq 1, \quad i \in I$,

with $\Gamma=\sqrt{\sum_{j \in J} \sigma_{j}^{2}}$. In (12) we still have the quadratic term $v_{i}^{2}$. Since $v_{i}^{2} \in[0,1]$ for all $i \in I$ (by definition), [26] proposed approximating $v_{i}^{2}$ for every $i \in I$ by a piecewise linear (convex) function which they model via integer programming using an ordered set of type 2 variables (SOS2) that they denote by $\left\{\lambda_{i 1}, \ldots, \lambda_{i n}\right\}$ with $n$ representing the number of sub-sets into which the interval $[0$, $1]$ is to be partitioned. We recall that in a SOS2 of non-negative variables, at most two such variables can be positive; moreover, if exactly two are positive then they must be consecutive in the ordered set [6]. The way of describing mathematically SOS2 variables is nowadays commonly known and thus we omit it here. Nevertheless, the interested reader can refer to [6] or [46] for further details.

If we denote by $b_{m}>0$ the $m$-th break point of interval $[0,1]$, $m \in\{1,2, ., n\}$ (with $b_{n}=1$ ) then, for every $i \in I, v_{i}$ and $v_{i}^{2}$ can be approximated by $\sum_{m=1}^{n} \lambda_{i m} b_{m}$ and $\sum_{m=1}^{n} \lambda_{i m} b_{m}^{2}$, respectively, with (i) $\sum_{m=1}^{n} \lambda_{\text {im }}=1$, (ii) $0 \leq \lambda_{i m} \leq 1$, and (iii) $\left(\lambda_{i 1}, \ldots, \lambda_{\text {in }}\right)$ being a SOS2. Constraints (10)-(13) can now be reformulated as follows:

$$
\begin{aligned}
& \sum_{j \in J} \frac{\mu_{j}}{\Gamma} y_{i j}+z_{1-\gamma_{i}} \sum_{m=1}^{n} \lambda_{i m} b_{m} \leq \frac{q_{i}}{\Gamma} x_{i}, \quad i \in I, \\
& \sum_{j \in J} \frac{\mu_{j}}{\Gamma} y_{i j}+z_{\zeta_{i}} \sum_{m=1}^{n} \lambda_{i m} b_{m} \geq \frac{\beta q_{i}}{\Gamma} x_{i}, \quad i \in I, \\
& \sum_{j \in J} \frac{\sigma_{j}^{2}}{\Gamma^{2}} y_{i j}=\sum_{m=1}^{n} \lambda_{i m} b_{m}^{2}, \quad i \in I, \\
& \sum_{m=1}^{n} \lambda_{i m}=x_{i}, \quad i \in I,
\end{aligned}
$$$$
\lambda_{\text {im }} \geq 0, \quad i \in I, m=1, \ldots, n .
$$

$\left(\lambda_{i 1}, \ldots, \lambda_{\text {in }}\right) \mathrm{SOS} 2, \quad i \in I$

In a standard integer programming formulation of SOS2 variables, the right-hand side of constraints (17) is usually 1 . However, in our case, we can enhance the model by considering $x_{i}$. We also note that constraints (14) together with (17) imply (9) (they ensure that $y_{i j}=0$ when $x_{i}=0$ ). Summing up, we proceed with our study by considering the model:

minimize

(1),

subject to (2), (4), (5), (8), (14) - (19).

\section{Multi-criteria approaches}

As we mentioned in Section 1, the objective function $f(\mathbf{x}, \mathbf{y})$ often considered in single-source facility location problems is the total cost for opening facilities and serving the customers (see, e.g., $[10,12,16,20,23,24])$. Even when stochastic demands have been considered, authors have assumed so [2,3].

Specific applications/problems may call for the use of other objective functions. Eiselt and Laporte [17] provide a classification of objectives of relevance in the context of facility location problems. This is highlighted by [47], who revisit those objectives. When it comes to such applications we find several examples. For instance, equitable response time is often an objective when locating emergency services (see, for instance, [42] and [38]). Marsh and Schiling [30] revisit different models for capturing equitable distribution of customers to facilities. Erkut et al. [18] investigate a multi-criteria facility location problem in the context of solid waste management. The authors consider economic and environmental criteria such as the greenhouse effect and energy consumption.

More recently, [25] and [26] discuss a single-source facility location problem emerging in the context of preventive humanitarian logistics: the shelter site location problem. They focus on objectives other than cost-oriented ones. Since these objectives are at the core of our case study, we specify them in detail.

In the shelter site location problem, each potential location for a shelter is given a weight previously computed using data corresponding to different aspects related with each location. These aspects help determining the "suitability" of potential locations and include terrain slope, distance to health institutions, soil type, electrical infrastructure, sanitary system, ownership status, et cetera. The goal is to select the locations for sheltering in such a way that the minimum weight among the open facilities is maximized. This represents, in fact, a so-called Rawlsian objective ${ }^{2}$ that is usually imposed by the organizations leading the process of building

\footnotetext{
2 Taking after the 20th century American philosopher John Rawls and based upon his notable ideas on justice as fairness stated in his work, entitled "A Theory of Justice", published in 1971.
} 
shelters. In such a case, the objective function to consider (and to be maximized) is $f(\mathbf{x}, \mathbf{y})=W_{\min }=\min _{i \in I \mid x_{i}=1}\left\{w_{i}\right\}$, where $w_{i}$ represents the weight of location $i \in I$. This is a maxmin non-linear objective function that can be linearized straightforwardly.

An alternative objective function is briefly mentioned by [26] but not considered explicitly in the models presented in that paper: the average weight of the open facilities. This is an objective function that also makes use of the weights $w_{i}, i \in I$. It is formally defined as

$$
f(\mathbf{x}, \mathbf{y})=W_{\mathrm{avg}}=\frac{\sum_{i \in I} w_{i} x_{i}}{\sum_{i \in I} x_{i}} \quad \text { (to be maximized). }
$$

This particular objective is not novel (see for instance [14]); however, its use in a multi-criteria context along with a Rawlsian objective is. On the other hand, linearizing this objective is not as straightforward as it is for $W_{\min }$. Nevertheless, it can be done by defining a new set of nonnegative auxiliary variables. For $i \in I$ we define $\tau_{i}=W_{\text {avg }} \times x_{i}$. By summing in $i \in I$ we obtain

$\sum_{i \in I} \tau_{i}=W_{\mathrm{avg}} \times \sum_{i \in I} x_{i}$

Using a set of appropriate constraints we can eventually ensure that $W_{\text {avg }}$ represents, in fact, the average of the weights of the selected facilities by using the following constraints to linearize constraint (20) [46]:

$\tau_{i} \leq \overline{W_{\text {avg }}} \times x_{i}, \quad i \in I$

$\tau_{i} \leq W_{\text {avg }}, \quad i \in I$

$\tau_{i} \geq W_{\text {avg }}-\left[\left(1-x_{i}\right) \times \overline{W_{\text {avg }}}\right], \quad i \in I$

$\sum_{i \in I} \tau_{i}=\sum_{i \in I}\left(w_{i} \times x_{i}\right)$

$\tau_{i} \geq 0, \quad i \in I$,

$W_{\text {avg }} \geq 0$.

In the above constraints, $\overline{W_{\text {avg }}}$ denotes the upper bound for $W_{\text {avg. }}$ In the shelter site location problem it can be set equal to 1 . Constraint (21) ensures that $\tau_{i}$ is equal to 0 when the corresponding $x_{i}$ is 0 . Constraints (22) and (23) ensure that $\tau_{i}$ equals to $W_{\text {avg }}$ when $x_{i}$ is equal to 1 . Constraint (24) is the linear representation of constraint (20) which is obtained by replacing $W_{\text {avg }}$ according to its definition provided before. The rest of the constraints are the domain constraints for the new variables $\tau_{i}$ and $W_{\text {avg }}$.

Finally, we refer a third objective of relevance in some applications: minimizing the average distance traveled to a facility. This is a relevant objective to consider in single-source facility location problems when the closest assignment constraints are considered. In fact, when the facilities have a limited capacity, the satisfaction of those constraints does not necessarily mean that the total traveled distance is minimized. As before, denote by $\ell_{i j}$ the distance between candidate facility location $i$ and demand point $j$; then the average distance traveled per person can be defined as:

$f(\mathbf{x}, \mathbf{y})=$ Average Distance Traveled $(\mathrm{ADT})=\frac{\sum_{i \in I, j \in J} \ell_{i j} d_{j} y_{i j}}{\sum_{j \in J} d_{j}}$.

The fact that several objective functions can be considered within the context of single-source facility location problems raises a question: is a single objective function selected among those ones enough to capture the goals of a decision maker? If not, then, a multi-criteria setting becomes more appropriate. As mentioned in Section 1, not much work can be found in terms of multicriteria discrete facility location problems and let alone when it comes to problems with single sourcing. Next, we fulfill this gap by considering multiple objectives in a chance-constrained single-source discrete facility location problem. We study two wellknown paradigms in multi-criteria optimization: vectorial optimization and goal programming.

\subsection{Vectorial optimization}

Suppose that we have $L$ objective functions of interest, denoted by $f_{\ell}(\mathbf{x}, \mathbf{y}), \ell=1, \ldots, L$. If no hierarchy is established between the objectives then the problem can be formulated as a vectorial optimization problem:

$\begin{array}{ll}\text { minimize } & f(\mathbf{x}, \mathbf{y})=\left(f_{1}(\mathbf{x}, \mathbf{y}), \ldots, f_{L}(\mathbf{x}, \mathbf{y})\right), \\ \text { subject to } & (2),(4),(5),(8),(14)-(18) .\end{array}$

It is well-known that in general there will be no single solution that simultaneously optimizes all objectives individually. This leads to replacing the concept of optimality by Pareto optimality or efficiency [21]. The main question becomes the determination of Pareto solutions.

Two popular methods for generating Pareto solutions in vectorial optimization problems are the weighting method and the $\varepsilon$ constraint method (see, e.g., [31]). We focus our attention on the latter due to the advantages it often has when compared with the former (the interested reader can refer to the above-mentioned reference for a deeper discussion).

In the $\varepsilon$-constraint method we optimize one objective function after setting the others as constraints (the so-called side objectives). The problem can be stated as follows (w.l.o.g.):

minimize $f_{1}(\mathbf{x}, \mathbf{y})$,

subject to $f_{\ell}(\mathbf{x}, \mathbf{y}) \leq \varepsilon_{\ell}, \quad \ell=2, \ldots, L$,

$$
(\mathbf{x}, \mathbf{y}) \in S \text {, }
$$

with $S$ denoting the feasibility set, i.e., the set of solutions $(\mathbf{x}, \mathbf{y})$ satisfying (2), (4), (5), (8), and (14)-(18). The Pareto solutions are obtained by performing a parametric variation in the vector of coefficients $\left(\varepsilon_{2}, \ldots, \varepsilon_{L}\right)^{T}$.

In the particular case of two objective functions (the most common in the location analysis literature), we can implement this method quite efficiently. In this case we have $f(\mathbf{x}, \mathbf{y})=$ $\left(f_{1}(\mathbf{x}, \mathbf{y}), f_{2}(\mathbf{x}, \mathbf{y})\right)$.

Denote by $f^{1}=\left(f_{1}^{1}, f_{2}^{1}\right)$ and $f^{2}=\left(f_{1}^{2}, f_{2}^{2}\right)$ two points in the criteria space such that $f_{1}^{1} \leq f_{1}^{2}$ and $f_{2}^{1} \leq f_{2}^{2}$. Using the terminology introduced by [8], we define by $R\left(f^{1}, f^{2}\right)$ the rectangle in the criteria space induced by $f^{1}$ and $f^{2}$.

A point $\bar{f}$ in the criteria space corresponding to a feasible solution with objective function values in $R\left(f^{1}, f^{2}\right)$ that corresponds to a solution with smallest value for $f_{2}(\mathbf{x}, \mathbf{y})$ among all solutions with smallest value for $f_{1}(\mathbf{x}, \mathbf{y})$, if it exists, is denoted by

$\bar{f}=\underset{(\mathbf{x}, \mathbf{y}) \in S}{\operatorname{lex} \min }\left\{f_{1}(\mathbf{x}, \mathbf{y}), f_{2}(\mathbf{x}, \mathbf{y}) \mid f(\mathbf{x}, \mathbf{y}) \in R\left(f^{1}, f^{2}\right)\right\}$

and can be determined by solving the sequence of optimization problems

$\bar{f}_{1}=\min _{(\mathbf{x}, \mathbf{y}) \in S}\left\{f_{1}(\mathbf{x}, \mathbf{y}) \mid f(\mathbf{x}, \mathbf{y}) \in R\left(f^{1}, f^{2}\right)\right\}$

and

$\bar{f}_{2}=\min _{(\mathbf{x}, \mathbf{y}) \in S}\left\{f_{2}(\mathbf{x}, \mathbf{y}) \mid f(\mathbf{x}, \mathbf{y}) \in R\left(f^{1}, f^{2}\right) \wedge f_{1}(\mathbf{x}, \mathbf{y}) \leq \bar{f}_{1}\right\}$.

Using the same terminology we can represent the process of finding a point in the criteria space corresponding to a feasible solution and with objective values in the rectangle $R\left(f^{1}, f^{2}\right)$ with 
smallest value for $f_{1}(\mathbf{x}, \mathbf{y})$ among all solutions with smallest value for $f_{2}(\mathbf{x}, \mathbf{y})$.

Assume that all the Pareto solutions (corresponding to the socalled efficient frontier in the criteria space) are sequenced nondecreasingly according to the values of the first objective function. The first and the last of such points are, respectively

$$
\begin{aligned}
& f^{*}=\underset{(\mathbf{x}, \mathbf{y}) \in S}{\operatorname{lex} \min }\left\{f_{1}(\mathbf{x}, \mathbf{y}), f_{2}(\mathbf{x}, \mathbf{y}) \mid f(\mathbf{x}, \mathbf{y})\right. \\
& \text { and } \\
& \left.f^{* *}=\operatorname{R}_{(\mathbf{x}, \mathbf{y}) \in S} \operatorname{lex}_{\min }\left\{f_{2}(\mathbf{x}, \mathbf{y}), f_{1}(\mathbf{x}, \mathbf{y}) \mid f(\mathbf{x}, \mathbf{y}),(-\infty, \infty)\right)\right\} \\
& \quad \in R((-\infty, \infty),(-\infty, \infty))\} .
\end{aligned}
$$

Now, all the efficient solutions can be obtained starting from $f^{*}$ and iteratively finding the non-dominated point that is closest to the last non-dominated point, say $f^{l}$, by solving

$\underset{(\mathbf{x}, \mathbf{y}) \in S}{\operatorname{lex} \min _{1}}\left\{f_{1}(\mathbf{x}, \mathbf{y}), f_{2}(\mathbf{x}, \mathbf{y}) \mid f(\mathbf{x}, \mathbf{y}) \in R\left(f^{l}-(0, \varepsilon), f^{* *}\right)\right\}$

with $\varepsilon$ denoting a small constant. This is done until $f^{* *}$ is reached. In Section 4 we present results obtained after applying this methodology to a specific problem.

For the three-objective case, the methodology for finding all the Pareto solutions is not as straightforward as the lexicographic one just revisited for the bi-objective case. In particular, an algorithmic approach is necessary for a successful and efficient implementation. In fact, it is well-known that the components of the $\varepsilon$-vector should be determined appropriately in order to ensure that the sequence of mono-objective problems defined by the application of the $\varepsilon$-constrained method allows finding all the Pareto solutions.

A particular case of interest for us is the one in which one of the objective functions can take values in a finite set of rather small cardinality. This idea was explored by [1] in the context of a relief item distribution problem in the event of a disaster. Those authors aimed at minimizing the total transportation time of the items, the number of first-aid workers required, and the non-covered demand among all affected areas. A three-criteria optimization problem was considered and a procedure for determining all the Pareto solutions was developed. Taking advantage of the fact that one of the objective functions takes integer values in a finite set, the authors choose as the single objective function to optimize one that takes fractional (continuous) values. The remaining objective functions induce two constraints. The integrality of one of the objective functions set as constraints makes it simpler to develop an iterative methodology for implementing $\varepsilon$-constraint method. In fact, all possible values of the one-sided objective are known in advance since they are finite (and they are just a few). Therefore, in the approach proposed in that work the authors set this objective function to its lowest possible value and perform the classical $\varepsilon$-constraint method for two objective functions as long as the model generates feasible solutions. When infeasibility is detected, the algorithm proceeds with the selection of the next value of the integer objective function. The process continues until all possible values of the integer objective function have been considered. The interested reader can refer to [1] for further details and for a detailed proof of their methods.

The above mentioned methodology can be applied even if one objective function can take fractional (continuous) values provided that only a finite number of values are possible. This is the case if we consider an objective function such $W_{\min }$. We elaborate on this idea later in Section 4.

\subsection{Goal programming}

When a hierarchy between the multiple objectives of interest is previously established by the decision maker, vectorial optimization is not the appropriate paradigm to consider for finding Pareto solutions. In that case, each objective function should be optimized only after the objective functions that are higher in the hierarchy have been optimized: a goal programming procedure emerges. The candidate optimal solutions in each level of the hierarchy are the multiple optimal solutions (if they exist) obtained in the previous levels.

Like in the previous section, we assume that there are $L$ objective functions of interest, denoted by $f_{\ell}(\mathbf{x}, \mathbf{y}), \ell=1, \ldots, L$. A goal programming model can be stated by assigning a different priority level to each goal. The priority levels are numbers in $\{1, \ldots, K\}$, with $K$ denoting the total number of goals. A goal typically involves the achievement (or failure by the smallest amount possible) of "target" values for one or several objective functions. Moreover, a goal is optimized only after all the previous goals in the hierarchy have been optimized. Accordingly, when we write $P_{k}\left[f_{k}\right]$ we are indicating that function $f_{k}$ should be the $k^{\text {th }}$ to be optimized. The problem can be written generically as follows:

$$
\begin{array}{rlr}
\text { minimize } & \sum_{k=1}^{K} P_{k}\left[\sum_{\ell=1}^{L} \alpha_{\ell k}^{+} d_{\ell}^{+}+\sum_{\ell=1}^{L} \alpha_{\ell k}^{-} d_{\ell}^{-}\right], & \\
\text {subject to } & (2),(4),(5),(18),(14)-(18), & \\
& f_{\ell}(\mathbf{x}, \mathbf{y})+d_{\ell}^{-}-d_{\ell}^{+}=G_{\ell}, & \\
& d_{\ell}^{-}, d_{\ell}^{+} \geq 0, & \ell=1, \ldots, L, \ldots, L .
\end{array}
$$

In this model, the overall objective function is conceptual since $P_{1}, \ldots, P_{K}$ are denoting priority levels; they represent neither a quantity nor a measure. This "objective function" indicates that first we optimize $\sum_{\ell=1}^{L} \alpha_{\ell 1}^{+} d_{\ell}^{+}+\sum_{\ell=1}^{L} \alpha_{\ell 1}^{-} d_{\ell}^{-}$; afterwards we optimize $\sum_{\ell=1}^{L} \alpha_{\ell 2}^{+} d_{\ell}^{+}+\sum_{\ell=1}^{L} \alpha_{\ell 2}^{-} d_{\ell}^{-}$in the set of multiple optimal solutions found for the first function; et cetera. The process stops either when the functions in all priority levels have been considered or when we reach a priority level for which multiple optima are no longer available. In this case, the single solution at hand is the optimal solution to the overall problem. In the above model, $G_{\ell}$ denotes the target value for objective function $f_{\ell}(\mathbf{x}, \mathbf{y}) ; d_{\ell}^{-}$and $d_{\ell}^{+}$denote the shortage and the surplus with respect to the target $(\ell=1, \ldots, L)$. Finally the coefficients $\alpha_{\ell k}^{+}$and $\alpha_{\ell k}^{-}$define the involvement (and its extent) of objective function $f_{\ell}(\mathbf{x}, \mathbf{y})$ in goal $k$.

The above model is interesting when one goal at a higher level in the hierarchy is prone to render multiple optimal solutions. This is exactly what happens in real world problems such as those discussed by [25] and [26] and that we will consider in the next section for illustrative purposes.

\section{A case study}

In order to show the relevance of the modeling aspects and procedures discussed in the previous sections, we consider a specific problem emerging in the context of humanitarian logistics: the shelter site location problem.

The handbook by the [41] emphasizes that having previously established shelter areas is crucial when it comes to disaster recovery. For the victims who lose their homes under some unfortunate event, it is critical to find a safe and secure shelter in which they can preserve their lives with dignity. The problem emerging in the preparedness phase for disaster relief that consists of choosing locations for sheltering is the so-called shelter site location problem and it has been studied by [25] and [26].

There are several features specific to the shelter site location problem that were considered in the aforementioned studies. First, candidate shelter locations are identified in advance and each of them is assigned a weight, which is a value in $[0,1]$. The weights are computed taking many aspects into account (see [25] for all the details). The candidate locations can be parks, yards, school gardens, parking lots, et cetera; i.e. a spot that can be character- 
ized as safe in the event of a disastrous situation. Second, there are service level requirements, which are related with capacity and minimum utilization rates for the shelters.

One specific case the authors are aware occurs in Turkey. In this country, the current methodology for selecting shelter areas considers as a primary objective the maximization of the minimum weight of open shelter areas [25]. As discussed before, this maxmin objective can be looked at as a Rawlsian approach to the problem since it targets fairness for the least advantaged victims of a disaster. This objective was introduced mathematically in Section $3\left(W_{\min }\right)$. In th current work, it is also chosen as the primary objective.

While raising the minimum weight of the selected shelter locations to the possible maximum level, the above Rawlsian objective does not ensure that the best-weighted locations are utilized although this is of relevance in the shelter site location problem. In other words, there may be alternative optima w.r.t. $W_{\text {min }}$ but with a different value for the average weight across the selected shel-

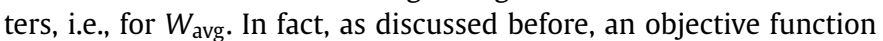
such as $W_{\min }$ is prone to generate plateaus in the objective space. In other words, one can easily obtain multiple optimal solutions when considering that measure alone. This provides strong evidence that by considering only a Rawlsian perspective we may obtain solutions in which the available resources are not used in the best way. Hence, a second objective (maximizing $W_{\text {avg }}$ ) emerges as relevant for ensuring a better public welfare.

Finally, when considering an optimization model for supporting decision making in the shelter site location problem, the closest assignment of populations to shelters is a key constraint to consider so that the models can "mimic" the behavior of people moving towards open facilities. Although these constraints aim at achieving a desirable outcome, they may not guarantee the best solution in terms of total distance traveled, which decreases its applicability. Similarly, it is easy to see that by only minimizing the total distance we cannot guarantee the closest assignment of the victims to the open shelters. Accordingly, another important objective to consider is the minimization of the average distance traveled-ADT (Recall the definition introduced in Section 3).

In synthesis, the three measures introduced in Section 3 are of great relevance in an application such as the shelter site location problem. Hence, we proceed with the analysis of our case study by considering the three objectives induced by those measures.

\subsection{Modeling specifications}

The general formulation presented in Section 2 can be specified for the single (Rawlsian) objective chance-constrained problem. Such specification leads to the optimization model that is introduced by [26]:

maximize $W_{\min }$,

subject to

$$
\begin{aligned}
& W_{\min } \leq w_{i} x_{i}+\left(1-x_{i}\right), \quad i \in I, \\
& (2),(4),(5),(8),(14)-(18) .
\end{aligned}
$$

In the above model it is assumed that $w_{i} \in[0,1], i \in I$. This formulation poses one difficulty if we wish to consider the objective function $W_{\min }$ within a vectorial optimization modeling framework. In fact, if a lexicographic approach such as the one described in Section 3 is considered, the objective function (27) will be represented as a constraint in some iterations $\left(W_{\min }=W_{\min }^{*}\right)$ ensuring that the value of $W_{\min }$ does not deteriorate from $W_{\min }^{*}\left(W_{\min }^{*}\right.$ represents the optimal solution of the counterpart model solved in the previous iteration with objective function (27)). In this case, constraint set (28) is not enough to ensure that the outcome in terms of $W_{\text {min }}$ represents, in fact, the minimum weight across the selected shelters. In other words, we may have an inconsistency in terms of the meaning of $W_{\text {min }}$ and its actual value produced by a lexicographic approach. What is more, this may lead to skipping some potential non-dominated solutions. These issues can be prevented by including a few additional constraints and a set of auxiliary binary variables denoted by $a_{i},(i \in I)$, as follows:

$W_{\min }=\sum_{i \in I}\left(w_{i} \cdot a_{i}\right)$

$\sum_{i \in I} a_{i}=1$

$a_{i} \leq x_{i}, \quad i \in I$,

$a_{i} \in\{0,1\}, \quad i \in I$.

Before presenting the data and the results for our case study, we emphasize that when dealing with $W_{\text {avg }}$, the constraints (21)(26) are appended to the corresponding models.

\subsection{Specialization of the $\boldsymbol{\varepsilon}$-constraint method for the 3-criteria stochastic shelter site location problem}

In Section 3 we pointed out that the $\varepsilon$-constraint method consists of solving a sequence of single objective problems considering one of the objective functions and incorporating the other ones (the side objectives) as constraints. We also mentioned the paper by [1] where an exact approach is proposed for finding all Pareto solutions in a 3-criteria problem when one of the objective functions takes values in a finite set (of small cardinality). Next, we adapt those ideas to our 3-criteria shelter site location problem.

In our problem we are considering the following three objective functions:

$f_{1}(\mathbf{x}, \mathbf{y}):$ ADT (minimize);

$f_{2}(\mathbf{x}, \mathbf{y}): W_{\text {avg }}$ (maximize);

$f_{3}(\mathbf{x}, \mathbf{y}): W_{\min }$ (maximize).

$f_{3}$ can take values in a finite set (whose cardinality is at most that of $I$ ). Hence, a single objective problem that we can consider for determining Pareto solutions is the following:

minimize $f_{1}(\mathbf{x}, \mathbf{y})$,

subject to $f_{2}(\mathbf{x}, \mathbf{y}) \geq \varepsilon_{2}$

$$
\begin{aligned}
& f_{3}(\mathbf{x}, \mathbf{y}) \geq \varepsilon_{3} \\
& (2),(4),(5),(8),(28), \\
& (14)-(18),(21)-(26),(29)-(32)
\end{aligned}
$$

We denote this problem by $P_{1}\left(\varepsilon_{2}, \varepsilon_{3}\right)$. We also consider a second problem to be used in our algorithmic approach, that we denote by $P_{2}\left(\varepsilon_{2}, \varepsilon_{3}\right)$, which results from replacing in $P_{1}\left(\varepsilon_{2}, \varepsilon_{3}\right)(34)$ by

$f_{3}(\mathbf{x}, \mathbf{y})=\varepsilon_{3}$.

Note that in constraints (33) and (34) we are using " $\geq$ " instead of " $\leq$ " because both objective functions $f_{2}(\mathbf{x}, \mathbf{y})$ and $f_{3}(\mathbf{x}, \mathbf{y})$ are to be maximized. Accordingly, some changes are necessary with respect to the "pure" minimization context considered in Section 3.

The proposed algorithm for finding all the Pareto solutions consists of two main stages. In the first one, we find all the nondominated and (weakly) dominated solutions. In the second stage, we iteratively eliminate the latter.

The first stage is detailed in Algorithm 1. In this algorithm, we denote by $\hat{f}_{1}, \hat{f}_{2}$, and $\hat{f}_{3}$ the current values of the objective functions considered. Recall that $|I|$ denotes the cardinality of potential shelter sites (which implies the maximum number of distinct 


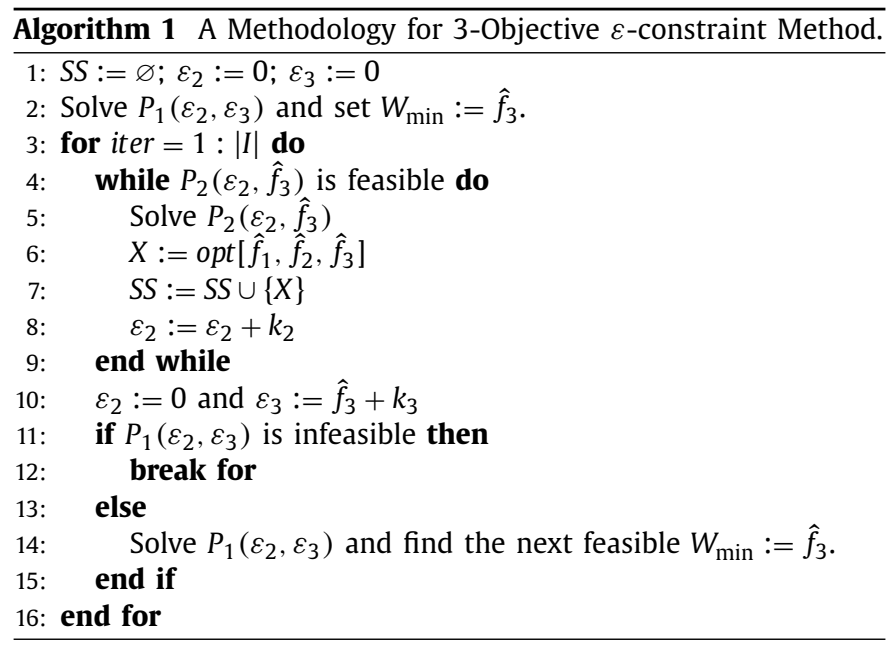

weight values). Furthermore, we denote by opt $\left[\hat{f}_{1}, \hat{f}_{2}, \hat{f}_{3}\right]$ the optimal solution to the current model $P_{2}\left(\varepsilon_{2}, f^{3}\right)$. Finally, $S S$ denotes the solution set (to be obtained by the execution of the algorithm).

We first solve the model $P_{1}(0,0)$ which will produce an initial value for $W_{\min }$ (line 2 ). Even though $W_{\min }$ can take values from a discrete set, it is not necessary to start from the lowest possible value. In fact, $P_{1}(0,0)$ will yield the lowest such value for a non-dominated solution, i.e., all the values for $W_{\text {min }}$ smaller than the one obtained when solving $P_{1}(0,0)$ render either infeasible or dominated solutions. In lines 4-9 of the algorithm, using $P_{2}\left(\varepsilon_{2}, \hat{f}_{3}\right)$, we fix $W_{\text {min }}$ and solve the model as if it is a biobjective one while increasing the second objective function value by $k_{2}$ until infeasibility is reached. In the meantime, we save the results in our solution set. Afterwards, we detect the next possible $W_{\text {min }}$ value by solving $P_{1}\left(0, \varepsilon_{3}\right)$ where $\varepsilon_{3}$ is assured to be strictly greater than the previous $W_{\min }$. The procedure is repeated until all the range of values for $W_{\text {min }}$ has been covered. Adequate values for the step sizes should result from a preliminary analysis performed using the specific data involved in an instance of the problem. For the data we considered in our study, we provide the details in Section 5.

This algorithm may produce (weakly) dominated solutions. These can be eliminated using a simple post processing procedure: a solution is compared with all other solutions; if it is associated with lower $W_{\min }$ and $W_{\text {avg }}$ values and higher distance value than some other solution, then it is removed from the solution set. The procedure is detailed in Algorithm 2. ParetoSet denotes the set of

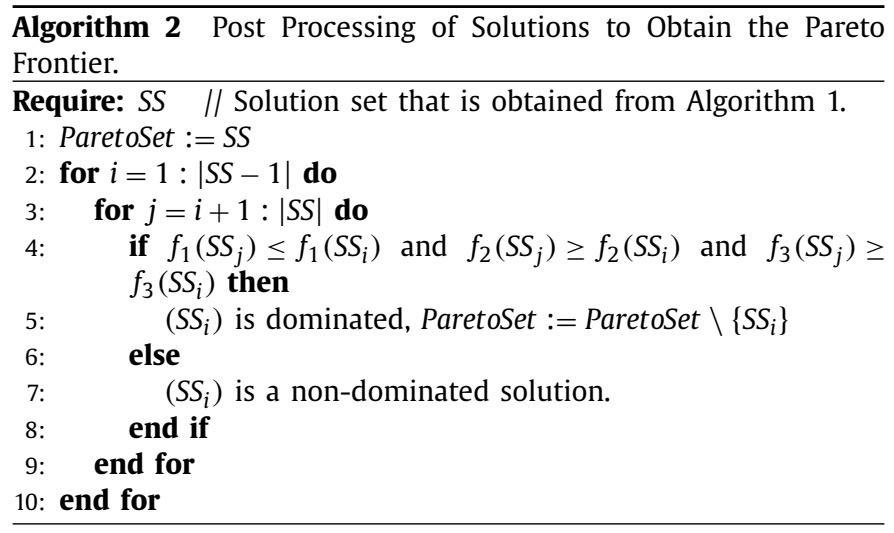

Pareto solutions obtained at the end of the whole procedure, and $S S_{i}$ is the $i$-th solution in set SS (the incumbent solution set).
The proof that the overall approach (Algorithm 1 and 2) provides the exact Pareto frontier for our problem follows exactly the same reasoning as the similar proof presented by [1] for their specific context.

Remark 1. In the above specialization of the $\varepsilon$-constrained method we chose ADT to be optimized (i.e., we set $f_{1}(\mathbf{x}, \mathbf{y})=$ ADT). We could have chosen $W_{\text {avg }}$ (making the necessary adjustments to Algorithm 1). The resulting Pareto front would, of course, be the same (we are determining all the Pareto solutions). However, a set of preliminary computations showed that minimizing the ADT results in significantly lower run times compared to maximizing $W_{\text {avg. }}$.

\subsection{Specialization of the goal programming model for the multi-criteria stochastic shelter site location problem}

As explained by [25] and [26] the existing quantitative approaches for the shelter site location problem call for a primary objective function to be optimized: $W_{\text {min }}$. If a decision maker looks at such objective as clearly more relevant than any other, then we should use an approach that enables us to solve the problem for that objective and then consider the set of (likely to exist) multiple optimal solutions to optimize other objectives. In other words, other objectives of interest can be optimized in the restricted set of solutions that contains the multiple optimal solutions to $W_{\min }$. This motivates the use of a goal programming approach. Using the terminology presented in Section 3.2 a goal programming model can be generically formulated for the shelter site location problem as follows (we consider without loss of generality that $W_{\text {avg }}$ has priority 2 and ADT priority 3):

$$
\begin{array}{ll}
\text { minimize } & P_{1}\left[d_{1}^{-}\right]+P_{2}\left[d_{2}^{-}\right]+P_{3}\left[d_{3}^{+}\right], \\
\text {subject to } & W_{\min }+d_{1}^{-}=w^{\mathrm{MAX}}, \\
& W_{\mathrm{avg}}+d_{2}^{-}=w^{\mathrm{MAX}}, \\
& A D T-d_{3}^{+}=0, \\
& (2),(4),(5),(8),(28), \\
& (14)-(18),(21)-(26),(29)-(32) \\
& d_{1}^{-}, d_{2}^{-}, d_{3}^{+} \geq 0
\end{array}
$$

In the above model, $w^{\mathrm{MAX}}$ denotes again the maximum value across all weights associated with the shelters. Moreover, the variables $d_{1}^{+}, d_{2}^{+}$and $d_{3}^{-}$(that according to the general model of Section 3.2 should be included in our model) are not being considered. We could have considered them but they are trivially equal to 0 due to the specification made for the goals. In fact, neither $W_{\text {min }}$ nor $W_{\text {avg }}$ can be greater than $w^{\mathrm{MAX}}$ and $A D T$ cannot be negative. In case we only wish to consider $W_{\text {min }}$ with one side objective we can simply set $P_{2}=0$ or $P_{3}=0$, depending on the objective we wish to exclude. In case we do not consider objective $W_{\text {avg }}$ we should also omit constraints (14)-(18).

\section{Computational experiments}

In this section, we consider two different data sets to test the multi-criteria chance-constrained modeling frameworks specified for our case study. We start by presenting the data we have worked with and then we analyze the results obtained.

\subsection{Data sets}

The first data set corresponds to Kartal, which is the 11th most populated district among the 39 districts of the metropolitan area of Istanbul. Kartal has more than 425,000 inhabitants and it is a $38.54 \mathrm{~km}^{2}$ district located near the western extension of the North 
Table 1

Used data sets' specifications.

\begin{tabular}{lll}
\hline & K45 & IST220 \\
\hline Number of candidate shelter location & 25 & 100 \\
Number of demand points & 20 & 120 \\
Minimum weight & 0.674 & 0.140633 \\
Maximum weight & 0.982 & 0.893454 \\
Mean weight & 0.827 & 0.575811 \\
Standard deviation of weights & 0.097 & 0.202467 \\
\hline
\end{tabular}

Anatolian fault. This area is considered to be a first degree earthquake threat zone which indicates its vulnerability against a future disaster.

The Kartal data set contains 45 relevant points that include the potential shelter locations and the possibly affected populations in case of an earthquake. This data was collected by [44] and has already been used in the literature ([25] and [26]). This data set will be referred as $K 45$ in the upcoming sections: "K" stands for Kartal and " 45 " for the total number of points involved in this set.

Istanbul is the world's 5th most crowded and 6th most densely populated city. It has $35 \%$ of its total population located in the east side of the Bosphorus, which is known as the Anatolian Side. The seismic research [36] shows that a catastrophic earthquake will inevitably hit the city in the near future. For this reason, in our study, we decided to consider the whole Anatolian side of Istanbul: it corresponds to our second data set. Naturally, it is significantly larger compared to K45. This data set will be referred to as IST220 in the upcoming sections: "IST" stands for Istanbul and "220" stands for the number of points involved in this set.

The specifications of the two data sets just introduced are given in Table 1 . In both cases, population data was obtained from the Turkish Statistical Institute.

In his Ph.D. thesis, [44] indicates that approximately $12.5 \%$ of the population of Istanbul would be in need for temporary housing if an earthquake occurs[25]. named this value as the Percent Affected Ratio $(P A R)$ and performed an extensive analysis with that specific percentage. However, [26] note that this parameter cannot be known in advance since it is dependent on many aspects of an uncertain-in-nature event; therefore, when shelter locations are being decided, demand variability should be accounted for. In this study, we have included such variability via the $P A R$ value using two different scenarios, both centered in the original value considered by [25] and [44]. In particular, we follow the same procedure introduced in [26]:

Scenario 1 - Low Variability: $P A R=0.125 \times U[0.95,1.05]$;

Scenario $2-$ High Variability: $P A R=0.125 \times U[0.85,1.15]$.

In the above scenarios, $U[a, b]$ denotes a pseudo-random random number generated according to a continuous uniform distribution in the interval $[a, b]$. For each scenario, 10 values were generated for PAR and each one was then multiplied by the number of inhabitants to obtain demand samples. From each such sample, $\mu_{j}$ and $\sigma_{j}^{2}$ are computed for all demand points $j \in J$ to be used in constraints (14)-(16).

In order to observe the behavior of the models proposed in the previous sections we also consider different values of $\beta$-the minimum allowed utilization rate of a shelter. The alternative values tested were $0.30,0.50$ and 0.70 . These alternatives lead to a varying size of the solution space, which enriches our analysis.

The chance constraint parameters $\gamma_{i}$ and $\zeta_{i}(i \in I)$ used in constraints (6) and (7) were set to 0.90 and 0.10 , respectively.

For both data sets, 10 breakpoints, $b_{1}=0.1, \ldots, b_{10}=1$, are used in the piecewise linear approximation considered for $v_{i}^{2}$ and represented by constraints (14)-(18). This means that the interval $[0,1]$ was always partitioned into 10 equal sub-intervals. In fact, a
Table 2

Step sizes to consider in the $\varepsilon$-constraint method.

\begin{tabular}{lll}
\hline Objective function & Step size K45 & Step size IST220 \\
\hline$W_{\text {min }}$ & $10^{-3}$ & $10^{-6}$ \\
$W_{\text {avg }}$ & $10^{-5}$ & $10^{-5}$ \\
$A D T$ & $10^{-3}$ & $10^{-3}$ \\
\hline
\end{tabular}

set of preliminary tests showed that this number ensures an accurate approximation for the value of $v_{i}^{2}$.

All of the models presented in this work were coded in Java API of CPLEX and solved using IBM CPLEX v12.6.1 that runs on a LinuX OS with 4xAMD Opteron Interlagos 6282SE 16 Core 2.6GHz 16MB L3 cache server processors with 96 GB of RAM.

\subsection{Results for 2-criteria vectorial optimization models}

In this section, we present the computational results obtained when considering a vectorial optimization modeling framework for the chance-constrained shelter site location problem we are investigating. We perform pairwise comparisons with our primary objective, $W_{\min }$.

While implementing the lexicographic approach, we need to define the step sizes for the transitions between consecutive iterations while not leaving out any non-dominated solutions (or to be adequately sensitive as required by the decision makers). It can be said that larger step size values speed up the computations of the models; whereas constitute a potential for overlooking some non-dominated solutions. Thus, it is essential to determine the step sizes so that they are sufficiently small to determine all solutions on the Pareto front and large enough to yield shorter computational times. To come up with suitable step size values for our case, we performed computational experiments with different values to find the most suitable ones. The weight data for candidate shelter sites of $K 45$ and IST220 data sets have 3 and 6 decimals, respectively. Therefore, since the smallest difference of $W_{\min }$ values between two solutions can be $10^{-3}$ and $10^{-6}$, we set these numbers to be the stepsize of $W_{\min }$ values for of $K 45$ and IST220. For the step size of the $W_{\text {avg }}$ measure, we started with $10^{-3}$ and changed it by the factor of 0.1 until $10^{-6}$. By the extensive computational analyses, we realized that $10^{-5}$ is sufficiently small for the step size of $W_{\text {avg }}$. Likewise, the stepsize of $A D T$ measure is set to be 1 meter $\left(10^{-3}\right.$ kilometers) for both data sets. The step size values for each measure and data set are shown on Table 2.

\subsubsection{Data set $\mathrm{K} 45$}

We look into Pareto solutions for two different bi-criteria problems: in the first one we aim at maximizing both $W_{\text {min }}$ and $W_{\text {avg }}$; in the second one we aim at maximizing $W_{\min }$ along with the minimization of ADT.

$\mathrm{W}_{\text {min }} v s . \mathrm{W}_{\text {avg. }}$ The results obtained when considering the objective functions $W_{\min }$ and $W_{\text {avg }}$ can be found in Table 3 . This table contains two sub-tables, each of which are for a different variability level. In each sub-table we distinguish the different values of $\beta$ analyzed. For each such value, we present a first line corresponding to $\max W_{\min }$. In this line, we observe the values obtained for the objective functions $W_{\min }$ and $W_{\text {avg }}$ when the single objective problem corresponding to maximizing $W_{\min }$ only was solved to optimality. Then, we present all the Pareto solutions obtained when a vectorial optimization modeling framework was considered involving both $W_{\text {min }}$ and $W_{\text {avg. }}$. We also provide the number of located shelters for each solution on a third column in each subtable headed by "\#".

Observing Table 3 we conclude that in all of the 6 combinations of $\beta$ and variability levels, the optimal solution to the single 
Table 3

\begin{tabular}{|c|c|c|c|c|}
\hline \multicolumn{5}{|c|}{ Scenario 1: Low variability } \\
\hline & & $W_{\min }$ & $W_{\text {avg }}$ & \# \\
\hline \multirow{3}{*}{$\beta=0.3$} & $\max W_{\min }$ & 0.847 & 0.89825 & 4 \\
\hline & Pareto solution 1 & 0.847 & 0.89825 & 4 \\
\hline & Pareto solution 2 & 0.827 & 0.93740 & 6 \\
\hline \multirow{3}{*}{$\beta=0.5$} & $\max W_{\min }$ & 0.847 & 0.88167 & 3 \\
\hline & Pareto solution 1 & 0.847 & 0.88167 & 3 \\
\hline & Pareto solution 2 & 0.827 & 0.9374 & 5 \\
\hline \multirow{2}{*}{$\beta=0.7$} & $\max W_{\min }$ & 0.847 & 0.88167 & 3 \\
\hline & Pareto solution 1 & 0.847 & 0.88167 & 3 \\
\hline \multicolumn{5}{|c|}{ Scenario 2: High variability } \\
\hline & & $W_{\min }$ & $W_{\text {avg }}$ & \# \\
\hline \multirow[t]{4}{*}{$\beta=0.3$} & $\max W_{\min }$ & 0.847 & 0.89825 & 4 \\
\hline & Pareto solution 1 & 0.847 & 0.89825 & 4 \\
\hline & Pareto solution 2 & 0.827 & 0.91360 & 5 \\
\hline & Pareto solution 3 & 0.809 & 0.93220 & 5 \\
\hline \multirow[t]{4}{*}{$\beta=0.5$} & $\max W_{\min }$ & 0.847 & 0.88167 & 3 \\
\hline & Pareto solution 1 & 0.847 & 0.88167 & 3 \\
\hline & Pareto solution 2 & 0.803 & 0.91560 & 4 \\
\hline & Pareto solution 3 & 0.801 & 0.9156 & 5 \\
\hline \multirow[t]{2}{*}{$\beta=0.7$} & $\max W_{\min }$ & 0.847 & 0.88167 & 3 \\
\hline & Pareto solution 1 & 0.847 & 0.88167 & 3 \\
\hline
\end{tabular}

objective problem ( $\max W_{\min }$ ) provides directly a Pareto solution (it always coincides with the Pareto solution 1). This can be explained by the fact that the data set under consideration is small and thus the number of Pareto solutions is itself small and not very "rich". This is confirmed by the fact that for the setting where the solution space is the smallest $(\beta=0.7)$, we only have one Pareto solution which is also provided by the single-objective solution for $W_{\min }$. We note also that not all the Pareto solutions obtained for the low variability setting are Pareto solutions in the high variability one and vice versa. It could also be observed that the number of located shelters tends to be larger for lower $\beta$ values and tends to increase when the $W_{\text {avg }}$ improves.

$\mathrm{W}_{\min }$ vs. ADT. For the second objective pairing, i.e., $W_{\min }$ and ADT, the results are provided in Table 4 . This table reads as Table 3.

Like observed when working with $W_{\text {min }}$ and $W_{\text {avg }}$ not all the Pareto solutions obtained for the low variability setting are Pareto solutions in the high variability one and vice versa. This indicates that capturing uncertainty (i.e., taking variability into account) is relevant since different solutions may be obtained .

Concerning the number of shelters selected, we can observe that it is quite similar to what was observed in Table 3.

Another interesting aspect is that, for $\beta=0.3$ (the less restricting value of $\beta$ ), the single objective solution corresponding to maximizing $W_{\min }$ is weakly dominated by the first Pareto solution in both variability scenarios. This shows the ability of our multicriteria framework to look for the alternative optimal solution for $W_{\text {min }}$ that minimizes ADT.

Observing Table 4 we also conclude that significant improvements can be achieved in ADT if we allow deteriorating the value of $W_{\text {min }}$ a little. Those improvements range between $28 \%$ and $50 \%$. Taking into account that ADT is an average value (per person), such improvements mean that the total distance traveled by all disaster victims can be significantly reduced in some cases. Hence, by using a multi-criteria approach thus obtaining a set of Pareto solutions we can provide the decision makers with a deeper insight concerning the trade-off between different objectives.

\subsubsection{Data set IST220}

The same scheme applied for K45 was also used for IST220 with the results reported next. This is a much larger data set and thus,
Table 4

Results for $K 45-W_{\min }$ vs. ADT.

\begin{tabular}{|c|c|c|c|c|}
\hline \multicolumn{5}{|c|}{ Scenario 1: Low Variability } \\
\hline & & $W_{\min }$ & ADT & \# \\
\hline \multirow[t]{5}{*}{$\beta=0.3$} & $\max W_{\min }$ & 0.847 & 2.983 & 4 \\
\hline & Pareto solution 1 & 0.847 & 2.911 & 4 \\
\hline & Pareto solution 2 & 0.827 & 1.698 & 5 \\
\hline & Pareto solution 3 & 0.801 & 1.572 & 7 \\
\hline & Pareto solution 4 & 0.694 & 1.500 & 8 \\
\hline \multirow[t]{4}{*}{$\beta=0.5$} & $\max W_{\min }$ & 0.847 & 3.068 & 3 \\
\hline & Pareto solution 1 & 0.847 & 3.068 & 3 \\
\hline & Pareto solution 2 & 0.827 & 1.728 & 5 \\
\hline & Pareto solution 3 & 0.694 & 1.671 & 7 \\
\hline \multirow[t]{3}{*}{$\beta=0.7$} & $\max W_{\min }$ & 0.847 & 3.068 & 3 \\
\hline & Pareto solution 1 & 0.847 & 3.068 & 3 \\
\hline & Pareto solution 2 & 0.827 & 1.789 & 5 \\
\hline \multicolumn{5}{|c|}{ Scenario 2: High Variability } \\
\hline & & $W_{\min }$ & ADT & \# \\
\hline \multirow[t]{6}{*}{$\beta=0.3$} & $\max W_{\min }$ & 0.847 & 3.068 & 3 \\
\hline & Pareto solution 1 & 0.847 & 2.911 & 3 \\
\hline & Pareto solution 2 & 0.809 & 2.077 & 6 \\
\hline & Pareto solution 3 & 0.803 & 1.990 & 6 \\
\hline & Pareto solution 4 & 0.801 & 1.532 & 7 \\
\hline & Pareto solution 5 & 0.694 & 1.505 & 8 \\
\hline \multirow[t]{5}{*}{$\beta=0.5$} & $\max W_{\min }$ & 0.847 & 3.068 & 3 \\
\hline & Pareto solution 1 & 0.847 & 3.068 & 3 \\
\hline & Pareto solution 2 & 0.809 & 2.435 & 4 \\
\hline & Pareto solution 3 & 0.803 & 1.211 & 4 \\
\hline & Pareto solution 4 & 0.801 & 1.974 & 5 \\
\hline \multirow[t]{5}{*}{$\beta=0.7$} & $\max W_{\min }$ & 0.847 & 3.068 & 3 \\
\hline & Pareto solution 1 & 0.847 & 3.068 & 3 \\
\hline & Pareto solution 2 & 0.809 & 2.732 & 4 \\
\hline & Pareto solution 3 & 0.801 & 2.424 & 4 \\
\hline & Pareto solution 4 & 0.674 & 2.217 & 5 \\
\hline
\end{tabular}

Table 5

Results for $I S T 220-W_{\min }$ vs. $W_{\text {avg }}$.

\begin{tabular}{|c|c|c|c|c|}
\hline \multicolumn{5}{|c|}{ Scenario 1: Low Variability } \\
\hline$\beta=0.3$ & & $W_{\min }$ & $W_{\text {avg }}$ & \# \\
\hline \multirow{5}{*}{$\beta=0.5$} & $\max W_{\min }$ & 0.595974 & 0.73458 & 12 \\
\hline & Pareto solution 1 & 0.595974 & 0.82540 & 10 \\
\hline & $\max W_{\min }$ & 0.595974 & 0.74345 & 9 \\
\hline & Pareto solution 1 & 0.595974 & 0.80902 & 7 \\
\hline & Pareto solution 2 & 0.580393 & 0.80996 & 7 \\
\hline \multirow[t]{3}{*}{$\beta=0.7$} & $\max W_{\min }$ & 0.595974 & 0.74225 & 5 \\
\hline & Pareto solution 1 & 0.595974 & 0.75822 & 6 \\
\hline & Pareto solution 2 & 0.580393 & 0.78775 & 6 \\
\hline \multicolumn{5}{|c|}{ Scenario 2: High Variability } \\
\hline \multirow[t]{3}{*}{$\beta=0.3$} & & $W_{\min }$ & $W_{\text {avg }}$ & $\#$ \\
\hline & $\max W_{\min }$ & 0.595974 & 0.74888 & 9 \\
\hline & Pareto solution 1 & 0.595974 & 0.82243 & 13 \\
\hline \multirow[t]{2}{*}{$\beta=0.5$} & $\max W_{\min }$ & 0.595974 & 0.72726 & 7 \\
\hline & Pareto solution 1 & 0.595974 & 0.80902 & 7 \\
\hline \multirow[t]{2}{*}{$\beta=0.7$} & $\max W_{\min }$ & 0.595974 & 0.75822 & 6 \\
\hline & Pareto solution 1 & 0.595974 & 0.75822 & 6 \\
\hline
\end{tabular}

we expect to observe the benefits of applying a multi-criteria modeling framework to the problem more explicitly.

$\mathrm{W}_{\min }$ vs. $\mathbf{W}_{\text {avg. }}$ The results for this setting can be observed in Table 5 that reads as the previous tables. The most prominent characteristic turns out to be the small number of Pareto solutions found. Nevertheless, maximizing $W_{\text {min }}$ or $W_{\text {avg }}$ individually does not necessarily lead to the same solution. This can be observed in more than one case. We recall that for each setting, the "last" Pareto solution maximizes $W_{\text {avg. }}$. Even though the number of Pareto solutions is considerably small, unlike the $K 45$ data set, among the 
Table 6

Results for $I S T 220-W_{\min }$ vs. ADT-low variability demand.

\begin{tabular}{lllll}
\multicolumn{4}{c}{ Scenario 1: Low Variability } \\
\hline & \multicolumn{2}{c}{$W_{\min }$} & ADT &
\end{tabular}

\begin{tabular}{|c|c|c|c|c|}
\hline \multicolumn{5}{|c|}{ Scenario 1: Low Variability } \\
\hline & & $W_{\min }$ & ADT & $\#$ \\
\hline & $\max W_{\min }$ & 0.595974 & 4.494 & 10 \\
\hline & Pareto solution 1 & $\overline{0} \overline{5} \overline{9} \overline{5} \overline{74}$ & $2 . \overline{2} \overline{4} \overline{7}$ & 6 \\
\hline & Pareto solution 2 & 0.580393 & 1.995 & 6 \\
\hline & Pareto solution 3 & 0.572222 & 1.739 & 4 \\
\hline$\stackrel{20}{102}$ & Pareto solution 4 & 0.502137 & 1.636 & 7 \\
\hline$\|$ & Pareto solution 5 & 0.471939 & 1.620 & 8 \\
\hline$\infty$ & Pareto solution 6 & 0.462376 & 1.575 & 7 \\
\hline & Pareto solution 7 & 0.417498 & 1.326 & 6 \\
\hline & Pareto solution 8 & 0.347547 & 1.135 & 7 \\
\hline & Pareto solution 9 & 0.268146 & 1.026 & 7 \\
\hline
\end{tabular}

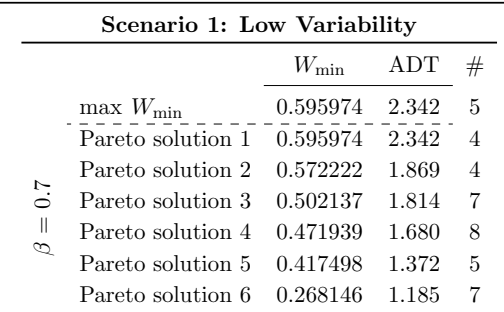
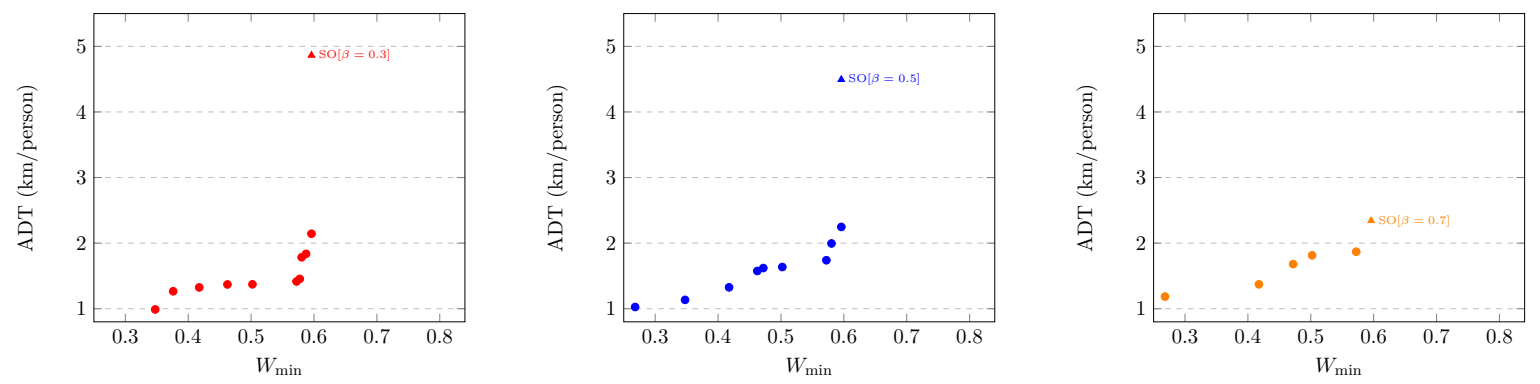

Table 7

Results for IST220- $W_{\min }$ vs. ADT-high variability demand.

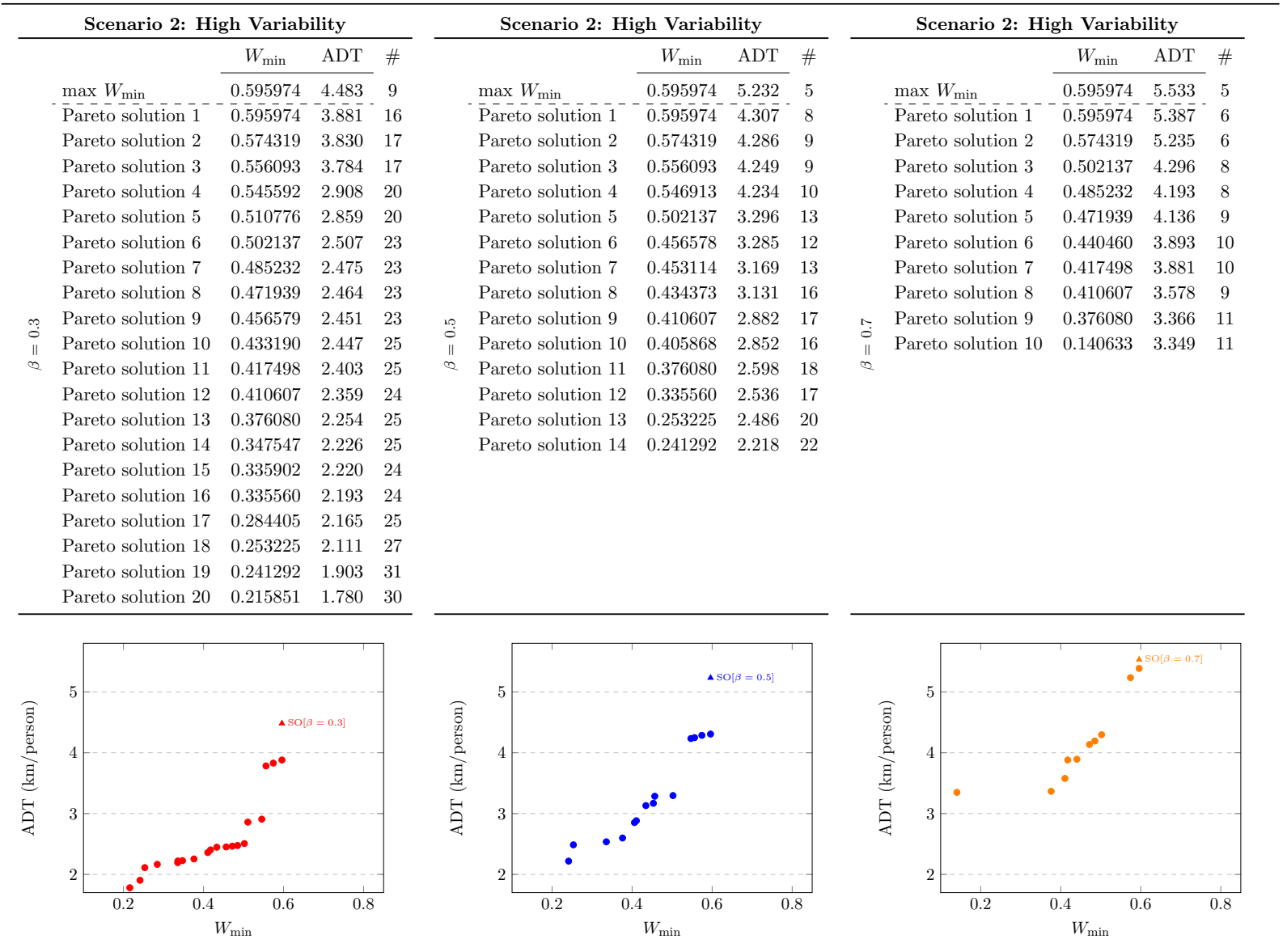


5 settings out of 6 , the first Pareto solution found immediately improves the objective function $W_{\text {avg }}$ value significantly when we compared to the single-objective solution that maximizes $W_{\min }$. In addition to those, on the contrary to what was observed on Table 3 and Table 4, one can notice that the multi-criteria framework is able to decrease the number of located shelters for two cases ( $\beta=0.3$ and $\beta=0.5$ for low variability). This implies that no generalization could be made on the relation between number of located shelters and $W_{\text {avg }}$ improvement. Nevertheless, the trend for an increase in the number of shelters when $\beta$ decreases is still present.

$\mathrm{W}_{\min }$ vs. ADT. The results obtained when the objective functions considered are $W_{\min }$ and ADT are depicted in Tables 6 and 7. These tables read as the previous ones but each one is associated with one variability level (small and high, respectively). Since the number of Pareto solutions is considerably higher than before, a graphical representation of the results is also provided at the bottom of the tables.

For the low variability scenario, when $\beta$ is 0.3 and 0.5 the average distance is improved more than $50 \%$ when we move from the single objective solution corresponding to $W_{\min }$ to the first Pareto solution found. On the other hand, when $\beta$ is 0.7 the solution space seems to be considerably reduced and such changes are not immediately observed or else they are not significant. Also, for this value of $\beta$, fewer Pareto solutions exist.

Looking into the number of located shelters of the solutions for each value of $\beta$, we cannot observe a general decremental or incremental pattern. Interestingly, for $\beta=0.5$, all the Pareto solutions have fewer open shelters when compared to the solution rendered by the single objective model.

For the high variability setting, a similar pattern can be observed in terms of number of Pareto solutions whilst $\beta$ is increasing. However, the stochasticity effects are significant on the average distance values, specifically in the earlier iterations. Therefore, this is again another major indication that (Pareto) solutions can be highly affected by the demand variability. Additionally, the higher demand variability leads to an increase in the number of Pareto solutions found. Although we can observe improvements in terms of ADT in the initial iterations of three $\beta$ levels, the improvement rates are not as significant as the low variability scenario. Compared to what we observed in the low variability scenario, we observe a significant increase in the number of selected shelters in the Pareto solutions obtained. This is particularly true for the lower value of $\beta$. Furthermore, we realized that no Pareto solution has less shelters selected than the number obtained by the single scenario model. For both low and high variability settings, the results show that there may be several quite different trade-off solutions which gives strength to the need of considering a multicriteria model for obtaining such solutions. This is more evident in the large IST220 data set considered.

\subsection{Results for the 3-criteria vectorial optimization model}

We consider now the vectorial optimization problem induced by the three objectives identified as relevant for the shelter site location problem: $W_{\text {min }}, W_{\text {avg }}$, and ADT.

The preliminary analysis whose results led to the values presented in Table 2 can also be used for specifying the step sizes necessary to run the $\varepsilon$-constrained method. In fact, the values $k_{2}$ and $k_{3}$ required by Algorithm 1 are directly taken from that table.

Like for the bi-criteria cases, we first present the results for K45 and then for IST220.
Table 8

Results overview for K45-3-criteria model.

\begin{tabular}{lllll}
\hline & & $\begin{array}{l}\text { Number of } \\
\text { solutions found }\end{array}$ & $\begin{array}{l}\text { Number of } \\
\text { solutions in the } \\
\text { Pareto Front }\end{array}$ & $\begin{array}{l}\text { Solution } \\
\text { time }\end{array}$ \\
\hline Low variability & $\beta=0.3$ & 15 & 10 & $3.2 \mathrm{sec}$ \\
& $\beta=0.5$ & 7 & 4 & $1.5 \mathrm{sec}$ \\
High variability & $\beta=0.7$ & 2 & 2 & $0.6 \mathrm{sec}$ \\
& $\beta=0.3$ & 19 & 17 & $4.9 \mathrm{sec}$ \\
& $\beta=0.7$ & 5 & 5 & $1.6 \mathrm{sec}$ \\
& $\beta=0.7$ & 4 & $1.6 \mathrm{sec}$ \\
\hline
\end{tabular}

Table 9

Pareto Solutions for K45-3-criteria model.

\begin{tabular}{|c|c|c|c|c|c|}
\hline \multicolumn{6}{|c|}{ Scenario 1: Low Variability } \\
\hline & & $W_{\min }$ & $W_{\text {avg }}$ & ADT & \# \\
\hline \multirow[t]{10}{*}{$\beta=0.3$} & Pareto solution 1 & 0.694 & 0.86100 & 1.500 & 8 \\
\hline & Pareto solution 2 & 0.694 & 0.86400 & 1.505 & 8 \\
\hline & Pareto solution 3 & 0.801 & 0.90414 & 1.527 & 7 \\
\hline & Pareto solution 4 & 0.801 & 0.90757 & 1.532 & 7 \\
\hline & Pareto solution 5 & 0.801 & 0.91067 & 1.618 & 6 \\
\hline & Pareto solution 6 & 0.801 & 0.91467 & 1.623 & 6 \\
\hline & Pareto solution 7 & 0.827 & 0.92533 & 1.698 & 6 \\
\hline & Pareto solution 8 & 0.827 & 0.93740 & 1.789 & 5 \\
\hline & Pareto solution 9 & 0.847 & 0.89160 & 2.911 & 5 \\
\hline & Pareto solution 10 & 0.847 & 0.89825 & 2.921 & 4 \\
\hline \multirow[t]{4}{*}{$\beta=0.5$} & Pareto solution 1 & 0.694 & 0.87300 & 1.671 & 7 \\
\hline & Pareto solution 2 & 0.827 & 0.89983 & 1.728 & 6 \\
\hline & Pareto solution 3 & 0.827 & 0.93740 & 1.789 & 5 \\
\hline & Pareto solution 4 & 0.847 & 0.88167 & 3.068 & 3 \\
\hline \multirow[t]{2}{*}{$\beta=0.7$} & Pareto solution 1 & 0.827 & 0.93740 & 1.789 & 5 \\
\hline & Pareto solution 2 & 0.847 & 0.88167 & 3.068 & 3 \\
\hline \multicolumn{6}{|c|}{ Scenario 2: High Variability } \\
\hline & & $W_{\min }$ & $W_{\text {avg }}$ & ADT & \# \\
\hline \multirow[t]{17}{*}{$\beta=0.3$} & Pareto solution 1 & 0.694 & 0.86400 & 1.505 & 8 \\
\hline & Pareto solution 2 & 0.801 & 0.90757 & 1.532 & 7 \\
\hline & Pareto solution 3 & 0.801 & 0.91467 & 1.623 & 6 \\
\hline & Pareto solution 4 & 0.801 & 0.92100 & 1.801 & 6 \\
\hline & Pareto solution 5 & 0.801 & 0.93220 & 1.893 & 5 \\
\hline & Pareto solution 6 & 0.803 & 0.87950 & 1.990 & 6 \\
\hline & Pareto solution 7 & 0.803 & 0.89883 & 2.004 & 6 \\
\hline & Pareto solution 8 & 0.803 & 0.90450 & 2.026 & 6 \\
\hline & Pareto solution 9 & 0.803 & 0.90560 & 2.096 & 5 \\
\hline & Pareto solution 10 & 0.803 & 0.91240 & 2.117 & 5 \\
\hline & Pareto solution 11 & 0.809 & 0.88050 & 2.077 & 6 \\
\hline & Pareto solution 12 & 0.809 & 0.89983 & 2.091 & 6 \\
\hline & Pareto solution 13 & 0.809 & 0.90550 & 2.112 & 6 \\
\hline & Pareto solution 14 & 0.809 & 0.90680 & 2.182 & 5 \\
\hline & Pareto solution 15 & 0.809 & 0.91360 & 2.204 & 5 \\
\hline & Pareto solution 16 & 0.847 & 0.89160 & 2.911 & 5 \\
\hline & Pareto solution 17 & 0.847 & 0.89825 & 2.921 & 4 \\
\hline \multirow[t]{5}{*}{$\beta=0.5$} & Pareto solution 1 & 0.801 & 0.85400 & 1.974 & 5 \\
\hline & Pareto solution 2 & 0.801 & 0.91560 & 2.060 & 5 \\
\hline & Pareto solution 3 & 0.803 & 0.91240 & 2.117 & 5 \\
\hline & Pareto solution 4 & 0.809 & 0.86350 & 2.435 & 4 \\
\hline & Pareto solution 5 & 0.847 & 0.88167 & 3.068 & 3 \\
\hline \multirow[t]{4}{*}{$\beta=0.7$} & Pareto solution 1 & 0.674 & 0.85040 & 2.217 & 5 \\
\hline & Pareto solution 2 & 0.801 & 0.86075 & 2.423 & 4 \\
\hline & Pareto solution 3 & 0.809 & 0.85825 & 2.732 & 4 \\
\hline & Pareto solution 4 & 0.847 & 0.88167 & 3.068 & 3 \\
\hline
\end{tabular}

\subsubsection{Data set $\mathrm{K} 45$}

In Table 8, we can observe algorithm specifications For this 45node instance, it can easily be noticed that the run times are small. Furthermore, it seems that the number of non-dominated solutions is slightly larger for the high variability scenario setting. In addition, the effect of the solution space size is observed on the gradual decrease in terms of Pareto solutions when we observe the increase of $\beta$. Table 9 shows the solutions defining the Pareto front for each setting. 
3D View

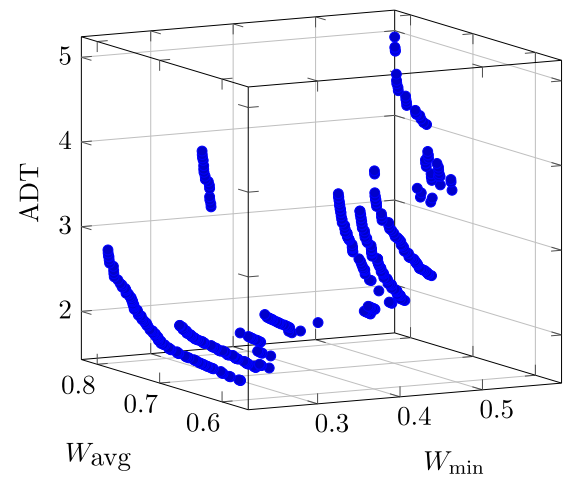

3D View

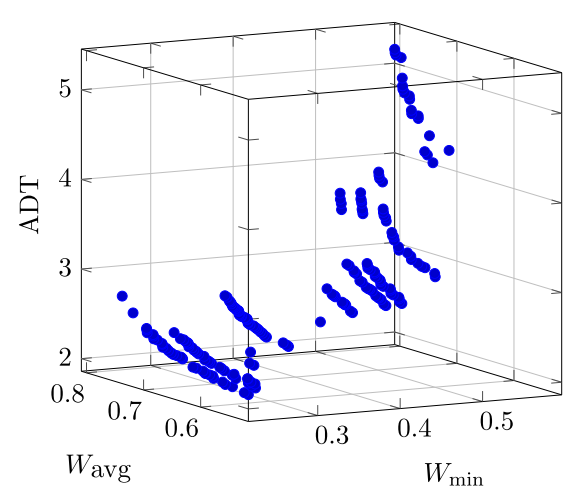

Front View

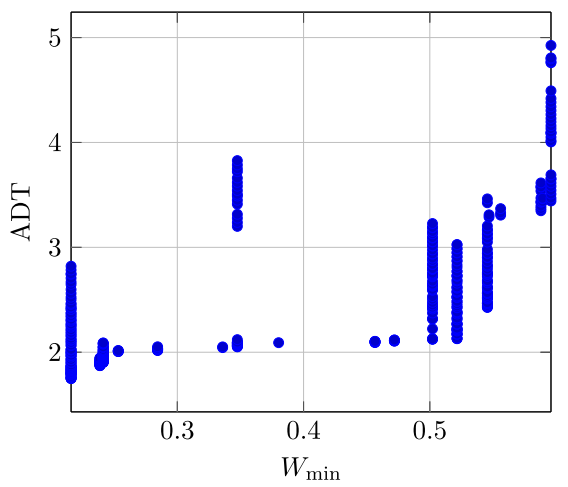

Fig. 1. Low variability $\& \beta=0.3$.

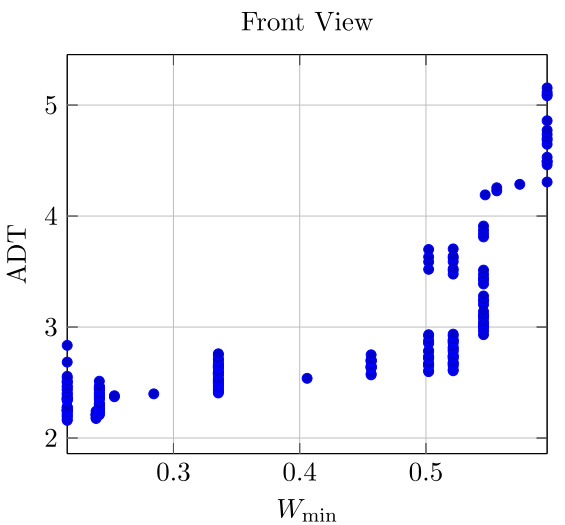

Fig. 2. Low variability $\& \beta=0.5$.
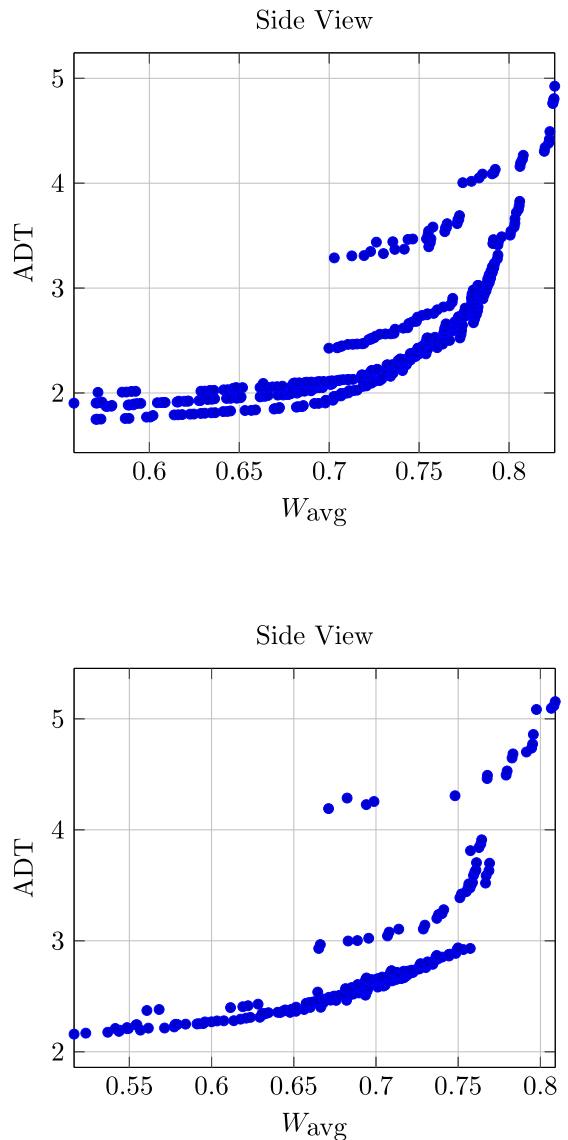

Table 10

Results overview for IST220-3-criteria model.

\begin{tabular}{lllll}
\hline & & $\begin{array}{l}\text { Number of } \\
\text { Solutions Found }\end{array}$ & $\begin{array}{l}\text { Number of } \\
\text { Solutions on } \\
\text { Pareto Front }\end{array}$ & $\begin{array}{l}\text { Solution } \\
\text { time }\end{array}$ \\
\hline Low Variability & $\beta=0.3$ & 1323 & 416 & $4 \mathrm{~h} 1 \mathrm{~m}$ \\
& $\beta=0.5$ & 462 & 209 & $3 \mathrm{~h} 8 \mathrm{~m}$ \\
& $\beta=0.7$ & 89 & 62 & $22 \mathrm{~m}$ \\
High Variability & $\beta=0.3$ & 1168 & 476 & $4 \mathrm{~h} \mathrm{3m}$ \\
& $\beta=0.5$ & 287 & 153 & $58 \mathrm{~m}$ \\
& $\beta=0.7$ & 40 & 28 & $8 \mathrm{~m}$ \\
\hline
\end{tabular}

\subsubsection{Data set IST220}

As expected, the number of iterations observed for IST220 is significantly higher than for $K 45$. Table 10 provides an overview of the results obtained. For $\beta=0.3$, the number of iterations turns out to be more than 1000 along with the total solution time slightly exceeding 4 hours for both uncertainty levels. With an increase of $\beta$ to 0.5 , we observe a decrease of more than $60 \%$ in the number of solutions while the number of Pareto solutions is dramatically decreased for high variability scenario as opposed to the nearly halved state of low variability one.

Since the number of solutions in the Pareto front is large, a tabular identification of such solutions is not informative. For this reason we depict those solutions graphically. Figs. 1-3 show the non-dominated solutions for the three $\beta$ levels and for the low uncertainty case. Likewise, Figs. 4-6 illustrate the Pareto solutions for the high variability case. For better understanding of the field of view in the 3-dimensional figures, front and side-views of the graphics are also provided.
Looking into these figures we observe that the solutions define a "cascade" pattern. This can be explained by the accumulation of solutions around a single value of $W_{\text {min }}$. Moreover, we can observe more scattered solutions when $\beta$ increases; i.e., the solutions seem to be more disjoint points than lines of points for higher $\beta$. As the number of Pareto solutions is scaled up for this 3-criteria setting (for IST220), it could be easily said that for a decision maker, the selection of a solution among these will not be as easy as in other settings. Nevertheless, a decision maker would certainly have a deeper insight concerning the trade-off between the different objectives considered.

\subsection{Results for the goal programming model}

The next set of results refers to the application of goal programming procedure to the multi-criteria shelter site location problem. Due to the practical relevance that optimizing $W_{\min }$ currently has for the institutions responsible for organizing sheltering (see [26]) we always consider that objective as the one with the highest priority. Then we study separately its combination with the other two objective functions. In other words, we present the results of our goal programming model considering (i) $W_{\text {min }}$ and $W_{\text {avg }}$; (ii) $W_{\text {min }}$ and ADT.

As before we present results for both K45 and IST220 data sets.

\subsection{1. $\mathbf{W}_{\min }$ Vs. $\mathbf{W}_{\text {avg }}$}

The obtained results are depicted in Table 11, where we always

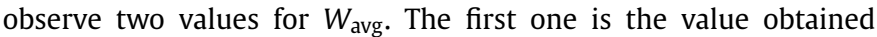
when optimizing $W_{\text {min }}$ only; the second one results from determining the best possible value of $W_{\text {avg }}$ when keeping $W_{\min }$ to the optimal value found. We can observe that for K45 the goal programming approach does not seem to be worth considering. In 
3D View

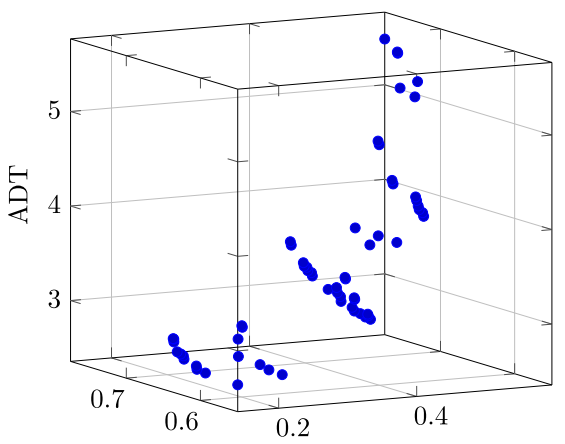

$W_{\text {avg }}$
Front View

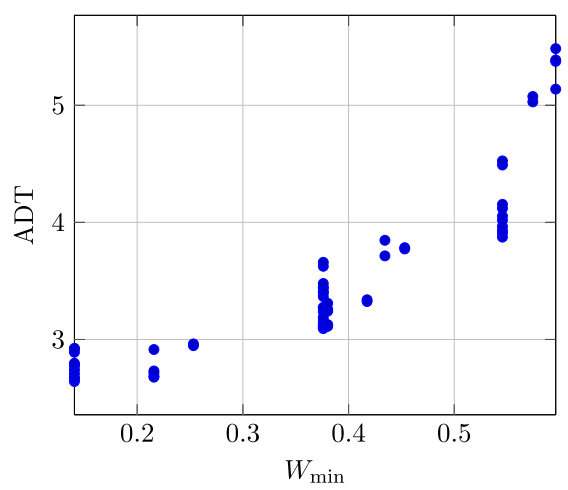

Fig. 3. Low variability $\& \beta=0.7$.

Front View

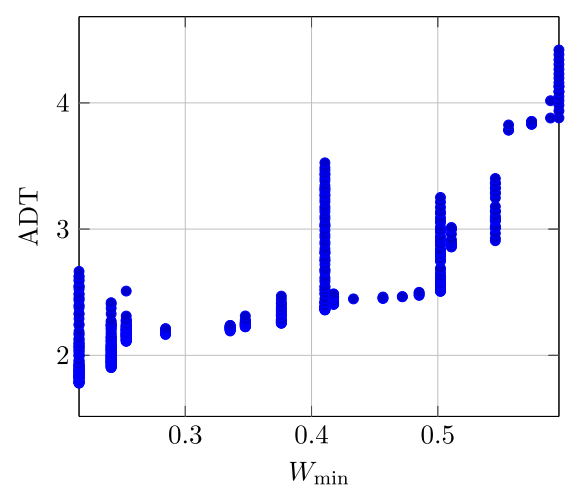

Fig. 4. High variability $\& \beta=0.3$.

Front View

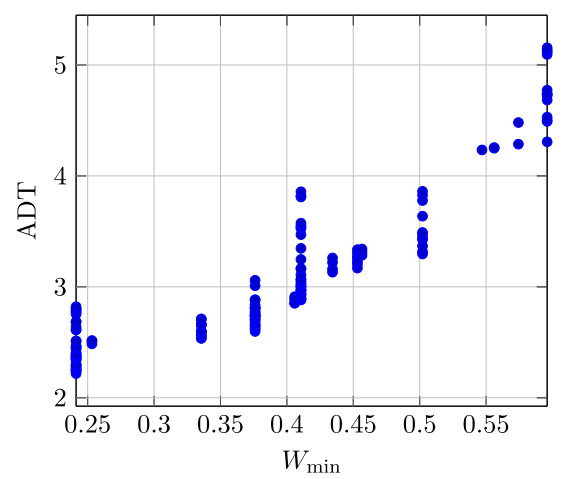

Fig. 5. High variability $\& \beta=0.5$.

Front View

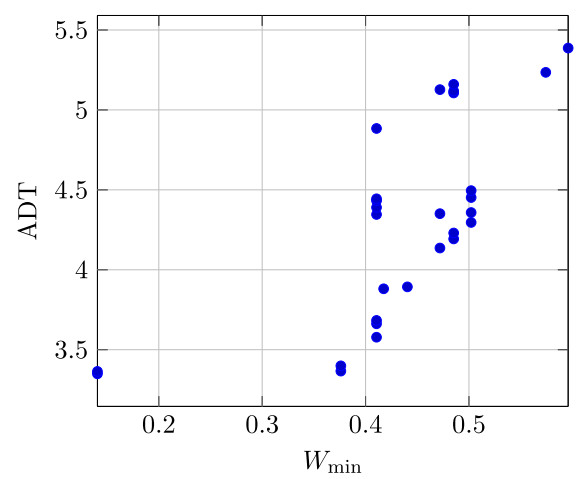

Fig. 6. High variability $\& \beta=0.7$.

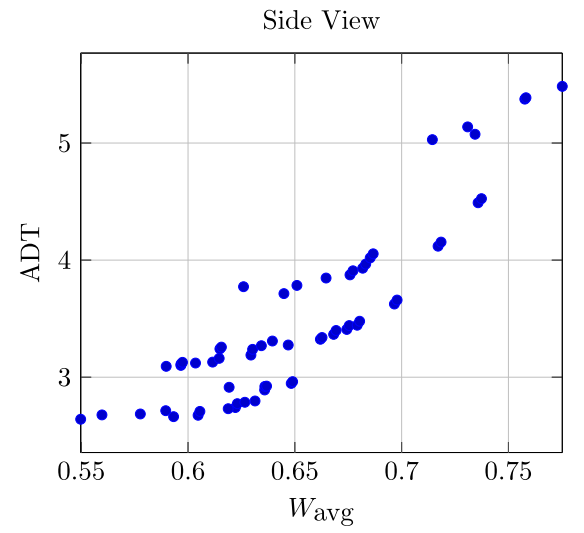

Side View

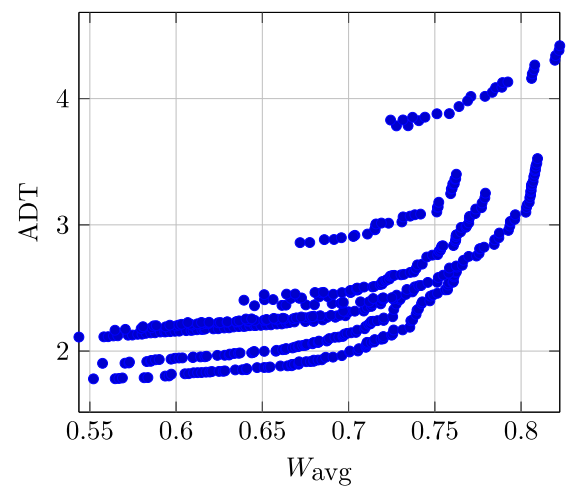

Side View

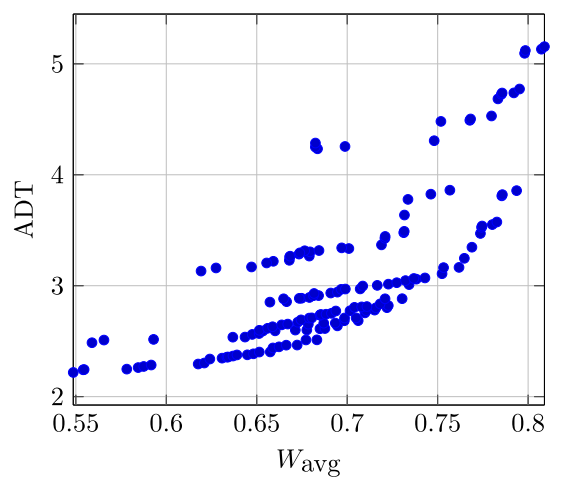

Side View

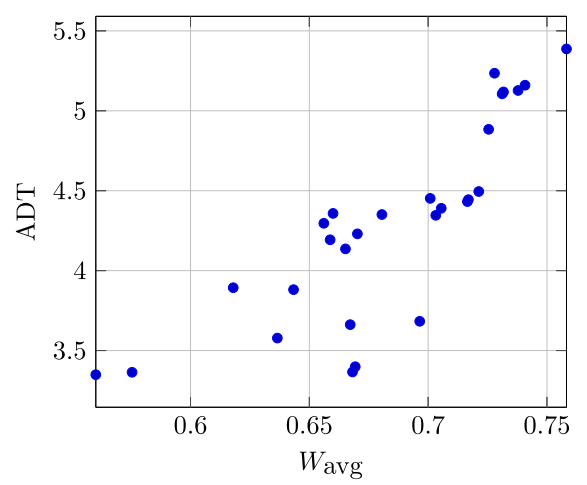

$W_{\text {avg }}$ $W_{\min }$

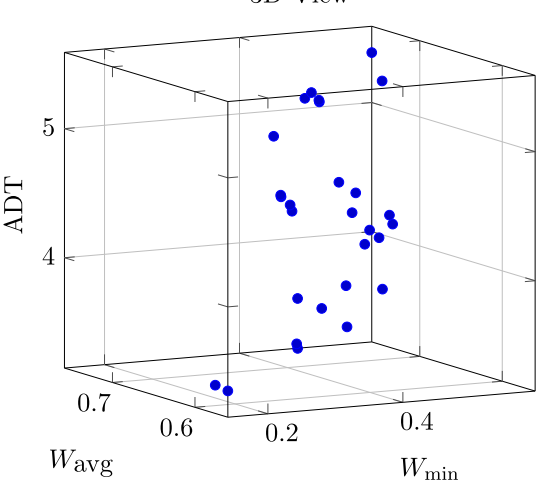


Table 11

Goal Programming results $-W_{\min }$ vs. $W_{\text {avg }}$.

\begin{tabular}{|c|c|c|c|c|c|}
\hline Data set & $\begin{array}{l}\text { Uncertainty } \\
\text { level }\end{array}$ & $\beta$ level & $W_{\min }$ & $W_{\mathrm{avg}}$ & Improvement \\
\hline K45 & Low & 0.3 & 0.847 & $\begin{array}{l}0.89825 \\
0.89825\end{array}$ & - \\
\hline K45 & High & 0.3 & 0.847 & $\begin{array}{l}0.89825 \\
0.89825\end{array}$ & - \\
\hline K45 & Low & 0.5 & 0.847 & $\begin{array}{l}0.88167 \\
0.88167\end{array}$ & - \\
\hline K45 & High & 0.5 & 0.847 & $\begin{array}{l}0.88167 \\
0.88167\end{array}$ & - \\
\hline K45 & Low & 0.7 & 0.847 & $\begin{array}{l}0.88167 \\
0.88167\end{array}$ & - \\
\hline K45 & High & 0.7 & 0.847 & $\begin{array}{l}0.88167 \\
0.88167\end{array}$ & - \\
\hline IST220 & Low & 0.3 & 0.595974 & $\begin{array}{l}0.73458 \\
0.82450\end{array}$ & $12.2 \%$ \\
\hline IST220 & High & 0.3 & 0.595974 & $\begin{array}{l}0.74888 \\
0.82243\end{array}$ & $9.8 \%$ \\
\hline IST220 & Low & 0.5 & 0.595974 & $\begin{array}{l}0.74345 \\
0.80902\end{array}$ & $8.8 \%$ \\
\hline IST220 & High & 0.5 & 0.595974 & $\begin{array}{l}0.72726 \\
0.80902\end{array}$ & $11.2 \%$ \\
\hline IST220 & Low & 0.7 & 0.595974 & $\begin{array}{l}0.74225 \\
0.75822\end{array}$ & $2.2 \%$ \\
\hline IST220 & High & 0.7 & 0.595974 & $\begin{array}{l}0.75822 \\
0.75822\end{array}$ & - \\
\hline
\end{tabular}

Table 12

GP results for $W_{\min }$ vs. Average Traveled Distance.

\begin{tabular}{|c|c|c|c|c|c|}
\hline Data Set & $\begin{array}{l}\text { Uncertainty } \\
\text { Level }\end{array}$ & $\beta$ Level & $W_{\min }$ & ADT & Improvement \\
\hline K45 & Low & 0.3 & 0.847 & $\begin{array}{l}2.983 \\
2.911\end{array}$ & $2.4 \%$ \\
\hline K45 & High & 0.3 & 0.847 & $\begin{array}{l}3.068 \\
2.911\end{array}$ & $5.1 \%$ \\
\hline K45 & Low & 0.5 & 0.847 & $\begin{array}{l}3.068 \\
3.068\end{array}$ & - \\
\hline K45 & High & 0.5 & 0.847 & $\begin{array}{l}3.068 \\
3.068\end{array}$ & - \\
\hline K45 & Low & 0.7 & 0.847 & $\begin{array}{l}3.068 \\
3.068\end{array}$ & - \\
\hline K45 & High & 0.7 & 0.847 & $\begin{array}{l}3.068 \\
3.068\end{array}$ & - \\
\hline IST220 & Low & 0.3 & 0.595974 & $\begin{array}{l}4.864 \\
2.143\end{array}$ & $55.9 \%$ \\
\hline IST220 & High & 0.3 & 0.595974 & $\begin{array}{l}4.483 \\
3.881\end{array}$ & $13.4 \%$ \\
\hline IST220 & Low & 0.5 & 0.595974 & $\begin{array}{l}4.494 \\
2.247\end{array}$ & $50.0 \%$ \\
\hline IST220 & High & 0.5 & 0.595974 & $\begin{array}{l}5.232 \\
4.307\end{array}$ & $17.7 \%$ \\
\hline IST220 & Low & 0.7 & 0.595974 & $\begin{array}{l}2.342 \\
2.342\end{array}$ & - \\
\hline IST220 & High & 0.7 & 0.595974 & $\begin{array}{l}5.533 \\
5.387\end{array}$ & $2.6 \%$ \\
\hline
\end{tabular}

fact, no improvement occurs in $W_{\text {avg. }}$. However, as the data set gets larger (IST220), we see the improvements achieved by implementing this approach, especially when $\beta=0.3$ and $\beta=0.5$. When $\beta$ is set to its maximum value, the benefits seem to diminish significantly.

\subsection{2. $\mathrm{W}_{\min }$ Vs. $A D T$}

The results obtained when considering $W_{\text {min }}$ and ADT in a goal programming modeling framework, are presented on Table 12 . We can clearly see that goal programming works extremely well, especially for IST220 when low uncertainty is assumed. This is a strong indication that the single-objective model can easily generate multiple optimal solutions thus providing room for improving ADT namely, when the solution space is larger. By using this bi-objective setting, we could easily detect the alternative solution with the same $W_{\min }$ value but an approximately $50 \%$ better distance value in certain cases.

\section{Conclusions}

In this work we proposed modeling frameworks for multicriteria chance-constrained discrete facility location problems with single sourcing. We considered two well-known paradigms in multi-criteria decision making: vectorial optimization and goal programming. We discussed ways of handling mathematically a set of probabilistic constraints that were included in the models.

We applied our modeling frameworks to the stochastic shelter site location problem using real data from Istanbul, Turkey. This required specializing existing methods and tools namely, when it comes to finding the exact Pareto front for 3-criteria vectorial optimization problems.

Our results show that the drawbacks of using only the Rawlsian approach can be smoothed by considering other objectives both when using a vectorial optimization modeling framework or goal programming.

The computational experiments highlight the importance of capturing uncertainty in the multi-criteria shelter site location problem. Nearly for all tested settings, it is observed that the number of efficient solutions found changes according to the demand variability. Moreover, the solutions themselves change according to the "degree" of uncertainty considered. This gives support to the claim that demand variability is worth considering in the decision making process as a way for providing resource management plans that can better hedged against uncertainty.

The computational experiments reported in this work show the effect of the size of the feasibility set on the relevance of considering a multi-criteria modeling framework. We could observe that such relevance is higher when large-scale data sets are used with "looser" constraint settings (e.g. service level constraints with lower $\beta$ values). Accordingly, by obtaining many efficient solutions, a decision maker can be provided with richer information for evaluating the trade-offs between different objectives and select the one that emerges as the most convenient or appealing.

One interesting aspect calling for further research would be the development of multi-criteria decision analysis tools such as analytical hierarchy process or multi-attribute value theory as a means for a rational selection of the best-fitting efficient solution among many. This would give a help to the decision maker in the search for the solutions that is closest to an "ideal preference".

Another research direction includes the development of algorithmic procedures for tackling larger instances of our problem, namely if a much larger number of scenarios is required for appropriately describing the underlying uncertainty.

\section{Acknowledgments}

This research has been partially supported by the Portuguese Science Foundation, project UID/MAT/04561/2013 (Centro de Matemática Aplicações Fundamentais e Investigação Operacional, Faculdade de Ciências da Universidade de Lisboa, Portugal) .

The authors would like to thank the three anonymous referees who have contributed in the further development of this manuscript by their suggestions and comments.

\section{References}

[1] Abounacer R, Rekik M, Renaud J. An exact solution approach for multi-objective location-transportation problem for disaster response. Comput Oper Res 2014;41:83-93.

[2] Albareda-Sambola M, Fernández E, Saldanha-da-Gama F. The facility location problem with Bernoulli demands. Omega (Westport) 2011;39:335-45. 
[3] Albareda-Sambola M, Fernández E, Saldanha-da-Gama F. Heuristic solutions to the facility location problem with general bernoulli demands. INFORMS J Comput 2017;29:737-53.

[4] Badri MA. Combining the analytic hierarchy process and goal programming for global facility location-allocation problem. Int J Prod Econ 1999;62:237-48.

[5] Barceló J, Casanovas J. A heuristic lagrangean algorithm for the capacitated plant location problem. Eur J Oper Res 1984;15:212-26.

[6] Beale EML, Tomlin JA. Special facilities in a general mathematical programming system for non-convex problems using ordered sets of variables. In: Lawrence J, editor. Proceedings of the Fifth International Conference on Operational Research. London: Tavistock Publications; 1970. p. 447-54.

[7] Bieniek M. A note on the facility location problem with stochastic demands. Omega (Westport) 2015;55:53-60.

[8] Boland N, Charkhgard H, Savelsbergh M. A criterion space search algorithm for biobjective integer programming: the balanced box method. INFORMS J Comput 2015;27:735-54.

[9] Castro J, Nasini S, Saldanha-da-Gama F. A cutting-plane approach for large-scale capacitated multi-period facility location using a specialized interior-point method. Math Progra 2017;163:411-44.

[10] Contreras IA, Díaz JA. Scatter search for the single source capacitated facility location problem. Ann Oper Res 2008;157:73-89.

[11] Correia I, Saldanha-da-Gama F. Facility location under uncertainty. In: Laporte G, Nickel S, Saldanha-da-Gama F, editors. Location Science. Berlin-Heidelberg: Springer; 2015. p. 177-203.

[12] Cortinhal MJ, Captivo ME. Upper and lower bounds for the single source capacitated location problem. Eur J Oper Res 2003;151:333-51.

[13] Costa MG, Captivo ME, Clímaco J. Capacitated single allocation hub location problem: a bi-criteria approach. Comput Oper Res 2008;35:3671-95.

[14] Current J, Min H, Schilling D. Multiobjective analysis of facility location decisions. Eur J Oper Res 1990;49:295-307.

[15] Dias J, Captivo ME, Clímaco J. An interactive procedure dedicated to a bicriteria plant location model. Comput Oper Res 2003;30:1977-2002.

[16] Díaz JA, Fernández E. A branch-and-price algorithm for the single source capacitated plant location problem. J Oper Res Soc 2002;53:728-40.

[17] Eiselt HA, Laporte G. Objectives in location problems. In: Drezner Z, editor. Facility Location: A survey of applications and methods. Berlin: Springer-Verlag; 1995. p. $151-80$.

[18] Erkut E, Karagiannidis A, Perkoulidis G, Tjandra S. A multicriteria facility location model for municipal solid waste management in north greece. Eur J Oper Res 2008;187(3):1402-21.

[19] Espejo I, Marín A, Rodríguez-Chía AM. Closest assignment constraints in discrete location problems. Eur J Oper Res 2012;219:49-58.

[20] Fernández E, Landete M. Fixed-charge facility location problems. In: Laporte G, Nickel S, Saldanha-da-Gama F, editors. Location Science. Berlin-Heidelberg: Springer; 2015. p. 47-77.

[21] Figueira J, Greco S, Ehrgott M. Multiple criteria decision analysis: state of the art surveys. Berlin-Heidelberg: Springer; 2016.

[22] Gouveia L, Saldanha-da-Gama F. On the capacitated concentrator location problem: a reformulation by discretization. Comput Oper Res 2006;33:1242-58.

[23] Guastaroba G, Speranza M. A heuristic for BILP problems: the single source capacitated facility location problem. Eur J Oper Res 2014;238:438-50.

[24] Holmberg K, Rönnqvist M, Yuan D. An exact algorithm for the capacitated facility location problems with single sourcing. Eur J Oper Res 1999;113:544-59.

[25] Kılcı F, Kara BY, Bozkaya B. Locating temporary shelter areas after an earthquake: a case for turkey. Eur J Oper Res 2015;243:323-32.
[26] Kınay OB, Kara BY, Saldanha-da-Gama F, Correia I. Modeling the shelter site location problem using chance-constraints: a case study for istanbul. Eur J Oper Res 2017. Under Review

[27] Klincewicz J, Luss H. A Lagrangian relaxation heuristic for capacitated facility location with single-source constraints. J Oper Res Soc 1986:495-500.

[28] Lin C. Stochastic single-source capacitated facility location model with service level requirements. Int J Prod Econ 2009;117:439-51.

[29] Maass KL. Mitigating hard capacity constraints in facility location modeling. University of Michigan; 2017. Ph.D. thesis.

[30] Marsh M, Schiling D. Equity measurement in facility location analysis: a review and framework. Eur J Oper Res 1994;74(1):1-17.

[31] Mavrotas G. Effective implementation of the $\varepsilon$-constraint method in multi-objective mathematical programming problems. Appl Math Comput 2009;213(2):455-65.

[32] Melo MT, Nickel S, Saldanha-da-Gama F. Dynamic multi-commodity capacitated facility location: a mathematical modeling framework for strategic supply chain planning. Comput Oper Res 2006;33:181-208.

[33] Melo MT, Nickel S, Saldanha-Da-Gama F. Facility location and supply chain management-a review. Eur J Oper Res 2009;196(2):401-12.

[34] Nickel S, Puerto J, Rodríguez-Chía AM. Multiple criteria decision analysis: state of the art surveys. In: Figueira J, Greco S, Ehrgott M, editors. International series in Operations Research and Management Science, 78. Berlin-Heidelberg: Springer; 2005. p. 761-87.

35] Nickel S, Puerto J, Rodríguez-Chía AM. Location problems with multiple criteria. In: Laporte G, Nickel S, Saldanha-da-Gama F, editors. Location Science. Berlin-Heidelberg: Springer; 2015. p. 205-47.

[36] Parsons T, Toda S, Stein RS, Barka A, Dieterich JH. Heightened odds of large earthquakes near istanbul: an interaction-based probability calculation. Science 2000;288(5466):661-5.

[37] Pirkul H. Efficient algorithms for the capacitated concentrator location problem. Comput Oper Res 1987;14:197-208.

[38] ReVelle C, Bigman D, Schilling D, Cohon J, Church R. Facility location: a review of context-free and ems models. Health Serv Res 1977;12(2):129-46.

[39] Rojeski P, ReVelle C. Central facilities location under an investment constraint. Geogr Anal 1970;2:343-60.

[40] Sinha SB, Sastry S. A goal programming model for facility location planning. Socioecon Plann Sci 1987;21:251-5.

[41] Sphere Project. Sphere Handbook: Humanitarian Charter and Minimum Standards in Disaster Response. 2011. Available at http://www.refworld.org/dodid/ 4ed8ae592.html [Accessed May 2017].

[42] Toregas C, Swain R, ReVelle C, Bergman L. The location of emergency service facilities. Oper Res 1971:19(6):1363-73.

[43] Trivedi A, Singh A. A hybrid multi-objective decision model for emergency shelter location-relocation projects using fuzzy analytic hierarchy process and goal programming approach. Int J Project Manage 2017;35(5):827-40.

[44] Unal G. A mathematical model based decision support system for emergency operations. Ph.D. Dissertation, Turkish Military Academy Defense Sciences Institutes; 2010

[45] Wagner JL, Falkson LM. The optimal nodal location of public facilities with price-sensitive demand. Geogr Anal 1975;7:69-83.

[46] Williams HP. Model building in mathematical programming. 5th. Chichester: Wiley; 2013.

[47] Zanjirani Farahani R, SteadieSeifi M, Asgari N. Multiple criteria facility location problems: a survey. Appl Math Model 2010;34:1689-709. 